TIT/HEP-618

\title{
Exact Results on the ABJM Fermi Gas
}

\author{
Yasuyuki Hatsuda*, Sanefumi Moriyamatt and Kazumi Okuyamatt \\ *Department of Physics, Tokyo Institute of Technology \\ Tokyo 152-8551, Japan \\ ${ }^{\dagger}$ Kobayashi Maskawa Institute $\& 3$ Graduate School of Mathematics, Nagoya University \\ Nagoya 464-8602, Japan \\ ${ }^{\ddagger}$ Department of Physics, Shinshu University \\ Matsumoto 390-8621, Japan
}

\begin{abstract}
We study the Fermi gas quantum mechanics associated to the ABJM matrix model. We develop the method to compute the grand partition function of the ABJM theory, and compute exactly the partition function $Z(N)$ up to $N=9$ when the Chern-Simons level $k=1$. We find that the eigenvalue problem of this quantum mechanical system is reduced to the diagonalization of a certain Hankel matrix. In reducing the number of integrations by commuting coordinates and momenta, we find an exact relation concerning the grand partition function, which is interesting on its own right and very helpful for determining the partition function. We also study the TBA-type integral equations that allow us to compute the grand partition function numerically. Surprisingly, all of our exact results of the partition functions are written in terms of polynomials of $\pi^{-1}$ with rational coefficients.
\end{abstract}

July 2012

\footnotetext{
*yhatsuda@th.phys.titech.ac.jp

${ }^{\dagger}$ moriyama@math.nagoya-u.ac.jp

${ }^{\ddagger}$ kazumi@azusa.shinshu-u.ac.jp
} 


\section{Introduction}

M-theory is expected to unify all of our understandings on the nonperturbative effects of string theory. Despite its importance, many aspects on this interesting theory await to be understood. One of the most mysterious facts on M-theory is the degrees of freedom of the branes. From the AdS/CFT correspondence, it is known [1] that $N$ coincident M2-branes (or M5-branes) have the degrees of freedom of order $N^{3 / 2}$ (or $N^{3}$, respectively). In the case of D-branes in string theory, the worldvolume theory can be described by the super Yang-Mills theory, which has the degrees of freedom $N^{2}$. Compared with such a case, the field theoretical realization on the branes in M-theory is still unclear.

Recently, there is a breakthrough [2] (along with its extension with fractional branes [3]) in understanding branes in M-theory, where it was proposed that the worldvolume theory of M2branes is described by the supersymmetric extension of the Chern-Simons theory. Concretely speaking, the proposal states that $N$ coincident M2-branes on the geometry $\mathbb{R}^{8} / \mathbb{Z}_{k}$ is described by the $\mathcal{N}=6$ Chern-Simons theory with gauge groups $U(N)_{k} \times U(N)_{-k}$ and bifundamental matters $A_{1,2}, B_{1,2}$ forming the superpotential $W=\frac{2 \pi}{k} \operatorname{Tr}\left(A_{1} B_{1} A_{2} B_{2}-A_{1} B_{2} A_{2} B_{1}\right)$ where the coupling constant is given by $g_{s}=\frac{2 \pi i}{k}$. The geometry $\mathbb{R}^{8} / \mathbb{Z}_{k}$ in a special case with $k=1$ is nothing but the flat space, hence it is natural to expect that the supersymmetry is enhanced to $\mathcal{N}=8$ in that case. It was then proposed that assuming the existence of the monopole operator we can construct the $\mathcal{N}=8$ supersymmetry algebra when $k=1,2$ [4, 5, 6, 7].

Subsequently, it was shown in [8] that the localization technique [9], which reduces the partition function defined by infinite-dimensional path integral to a finite-dimensional matrix model, is applicable to supersymmetric Chern-Simons theories. This matrix model has the measure of the Chern-Simons type and many techniques from the Chern-Simons matrix model such as [10] was utilized [11] to obtain not only the $N^{3 / 2}$ behavior of the free energy in the

planar limit but also the shift of 't Hooft coupling $\frac{N}{k} \rightarrow \frac{N}{k}-\frac{1}{24}$ as expected from the Euler coupling in the gravity dual [12].

Besides, it was also noticed that this matrix model is closely related to the topological string theory on a local Calabi-Yau over the Hirzebruch surface $\mathbb{F}_{0}=\mathbb{P}^{1} \times \mathbb{P}^{1}$. Using this relation, we can study the higher genus contribution by borrowing a celebrated recursive relation, the holomorphic anomaly equation [13], from the topological string theory. Following interesting observations by [14], it was found in [15] that we obtain the Airy function after summing up the contributions from all genus.

This result on the Airy function suggests some interesting relations to previous understanding on M-theory. In [16] it was noticed that the Airy function also appears in other context of M-theory as the wave-function of the universe. Further, the integral expression of 
the Airy function $\operatorname{Ai}(z)=\int_{C} d t \exp \left(-z t+\frac{t^{3}}{3}\right)$, which is a contour integral of an exponentiated cubic term, looks similar to the partition function $Z=\int D A \exp (-S[A])$ of the cubic Chern-Simons action $S[A]$. Therefore, we are led to expect a deep relation among M-theory, the Chern-Simons theory and the Airy function.

Another expectation from this result is its implication in understanding some of hidden structures in the membrane theory. String theory originates from the Veneziano amplitude in the dual resonance model, out of which the string worldsheet conformal symmetry was realized. Similarly, one might expect to find some hidden symmetry out of this Airy function and realize some structure for M-theory. An interesting hidden structure of this Airy function was beautifully realized by [17]. It was shown that the partition function of M2-branes (as well as its generalization with $\mathcal{N}=3$ supersymmetry) can be rewritten exactly into the partition function of a Fermi gas system with the density matrix (related to Hamiltonian by $\rho=e^{-H}$ )

$$
\rho=\frac{1}{\sqrt{2 \cosh \frac{q}{2}}} \frac{1}{2 \cosh \frac{p}{2}} \frac{1}{\sqrt{2 \cosh \frac{q}{2}}},
$$

and, with the standard analysis of the statistical mechanics, we can reproduce the behavior of $N^{3 / 2}$ and Airy function easily. Very recently, it was further proposed in [18] that we can generalize this formalism to the $\mathcal{N}=2$ case by introducing interactions to the Fermi particles and in [19] that we can extend to the cases with Wilson loops.

This simple rederivation of the Airy function implies the importance of this Fermi gas formulation. Since we have already a good control on the perturbative terms using the statistical mechanical method, as a next step, we would like to proceed to the study of this quantum mechanics extensively beyond the perturbation theory and to obtain some non-perturbative information out of it.

Aside from its possible important message on M-theory, the mathematical structure in this maximally supersymmetric theory seems very interesting. The supergravity analysis [20] suggests the BPS condition is expressed by a continuous Toda equation and the Hamiltonian of this Fermi gas system takes the form similar to the relativistic Toda theory [21, 22]. Therefore, it is natural to expect some kind of the exactly solvable structure in this theory. Some of the exact results was found in [23].

We would like to make a first step towards the goal to compute the exact partition function of the ABJM theory for arbitrary $N$ and arbitrary Chern-Simons level $k$, and to solve the eigenvalue equation of the corresponding Fermi gas system. Heading for this goal, we have found some interesting exact results on this theory as we will report in this paper.

As we will see in the next section, the (grand) partition function of this Fermi gas system is computed by the trace of the power of the density matrix. The main difficulty resides in 
the computation of convoluting the matrix element successively,

$$
\rho^{n}\left(q_{0}, q_{n}\right)=\int \frac{d q_{1}}{2 \pi} \frac{d q_{2}}{2 \pi} \cdots \frac{d q_{n-1}}{2 \pi} \rho\left(q_{0}, q_{1}\right) \rho\left(q_{1}, q_{2}\right) \cdots \rho\left(q_{n-1}, q_{n}\right) .
$$

Following the previous work on the thermodynamic Bethe ansatz (TBA) equations [24, 25], here we are able to express the matrix elements in terms of more basic vector quantities and reducing the computation of convoluting matrices into that of convoluting vectors.

In addition, we have also attempted to reduce the integration by commuting the coordinate $q$ and the momentum $p$. We have found that, although the naive commutation relation is anomalous, interestingly the additional term to the naive commutation relation can be expressed in terms of a projection operator $|p=0\rangle\langle q=0|$. Using this relation, we can express $\operatorname{Tr} \rho^{n}$ for odd $n$ by some combinations of $\left\langle q=0\left|(2 \cosh q / 2)^{-1 / 2} \rho^{m}(2 \cosh q / 2)^{1 / 2}\right| p=0\right\rangle$. For even $n$, we have found an interesting novel relation which separates the density matrix into two parities.

We will mostly restrict ourselves to the maximally supersymmetric case $k=1$. Most of our following analysis goes straightforwardly to other values of $k$ though a key relation will only look simple for $k=1$. The case $k=1$ is particularly interesting from the viewpoint of holography since the ABJM theory at $k=1$ is conjectured to be dual to the M-theory on $A d S_{4} \times S^{7}$ which is in the truly M-theoretic regime. The study of the ABJM matrix model in the large $N$, finite $k$ regime was initiated by [26] and further studied in [17] from the Fermi gas approach. However, the approach of [17] is restricted to the perturbation around $k=0$. We would like to study the $k=1$ case exactly.

As a result, we have computed the exact partition function $Z(N)$ up to $N=9$. We shall summarize our results in the following,

$$
\begin{array}{rlrl}
Z(1) & =\frac{1}{4}, & Z(2) & =\frac{1}{16 \pi}, \\
Z(3) & =\frac{\pi-3}{2^{6} \pi}, & Z(4) & =\frac{10-\pi^{2}}{2^{10} \pi^{2}}, \\
Z(5) & =\frac{-9 \pi^{2}+20 \pi+26}{2^{12} \pi^{2}}, & Z(6) & =\frac{36 \pi^{3}-121 \pi^{2}+78}{2^{14} \cdot 3^{2} \pi^{3}}, \\
Z(7) & =\frac{-75 \pi^{3}+193 \pi^{2}+174 \pi-126}{2^{16} \cdot 3 \pi^{3}}, & Z(8) & =\frac{1053 \pi^{4}-2016 \pi^{3}-4148 \pi^{2}+876}{2^{21} \cdot 3^{2} \pi^{4}}, \\
Z(9) & =\frac{5517 \pi^{4}-13480 \pi^{3}-15348 \pi^{2}+8880 \pi+4140}{2^{23} \cdot 3^{2} \pi^{4}} .
\end{array}
$$

Surprisingly, all of these results are written in terms of polynomials of $\pi^{-1}$ with rational coefficients.

This paper is organized as follows. In Section 2, we present our general procedure to compute the grand partition function. Applying the lemma of Tracy-Widom [24], we first 
show that the power of density matrix $\rho^{n}\left(q, q^{\prime}\right)$ is written in terms of a set of functions of one variable. We also show that the eigenvalue problem of $\rho$ is reduced to the diagonalization of a certain Hankel matrix. In Section 3, we find that the commutation relation of $\left(2 \cosh \frac{q}{2}\right)^{-1}$ and $\left(2 \cosh \frac{p}{2}\right)^{-1}$ appearing in $\rho$ contains an anomalous term, which can be expressed by a insertion of projection operator. Then we show that such anomalous commutation relation leads to a nontrivial relation of the grand partition function. In Section 4, using our general method, we compute exactly the first few terms of the Taylor expansion of the grand partition function at $k=1$. These are nothing but the partition functions $Z(N)$. Along the way, we find a non-trivial equation relating the even part and odd part of density matrix. In Section 5, some numerical results are given. We consider the TBA-type integral equation proposed in [25] to compute $\operatorname{Tr} \rho^{n}$. These integral equations are powerful in the numerical analysis. We also study the numerical solutions of the eigenvalue problem of the Fermi gas system. In Section 6, we compare our results with the known perturbative results of the ABJM matrix model and find a good agreement with the Airy function even for small $N$. We study the non-perturbative corrections to the free energy, and find numerical evidence that the leading correction scales as $e^{-2 \pi \sqrt{2 N}}$. We also consider the expectation value of $N$ in the grand canonical ensemble. We find that the numerical computation using TBA-like equations smoothly interpolates our exact results and the perturbative results. Finally, we conclude in Section 7 with discussions and future directions. Appendices A, B and C contain some details of the computations skipped in the text.

\section{Grand partition function of ABJM theory}

The aim of this section is to present a general prescription which is helpful for computing the grand partition function of the ABJM theory. The concrete computation based on the method given here will be presented in Section 4 .

Let us start by reviewing the Fermi gas formalism developed in [17]. By using the localization, the partition function of the ABJM theory on $S^{3}$ is written as the matrix integral

$$
Z(N)=\frac{1}{N !^{2}} \int \frac{d^{N} \mu}{(2 \pi)^{N}} \frac{d^{N} \nu}{(2 \pi)^{N}} \frac{\prod_{i<j}\left[2 \sinh \left(\frac{\mu_{i}-\mu_{j}}{2}\right)\right]^{2}\left[2 \sinh \left(\frac{\nu_{i}-\nu_{j}}{2}\right)\right]^{2}}{\prod_{i, j}\left[2 \cosh \left(\frac{\mu_{i}-\nu_{j}}{2}\right)\right]^{2}} \exp \left[\frac{i k}{4 \pi} \sum_{i=1}^{N}\left(\mu_{i}^{2}-\nu_{i}^{2}\right)\right] \text {. }
$$

Applying the Cauchy identity to the measure factor and performing the Gaussian integral over 
$\mu_{i}, \nu_{i}$, this partition function is recast into the "mirror" expression [27, 23, 17]

$$
Z(N)=\frac{1}{2^{N} N !} \int \frac{d^{N} q}{(2 \pi k)^{N}} \prod_{i} \frac{1}{2 \cosh \frac{q_{i}}{2}} \prod_{i<j} \tanh ^{2} \frac{q_{i}-q_{j}}{2 k} .
$$

The key idea of [17] is to rewrite it as the partition function of an ideal Fermi gas

$$
Z(N)=\frac{1}{N !} \sum_{\sigma \in S_{N}}(-1)^{\epsilon(\sigma)} \int \frac{d^{N} q}{(2 \pi k)^{N}} \prod_{i=1}^{N} \rho\left(q_{i}, q_{\sigma(i)}\right)
$$

where

$$
\rho\left(q_{1}, q_{2}\right)=\frac{1}{\left(2 \cosh \frac{q_{1}}{2}\right)^{1 / 2}} \cdot \frac{1}{2 \cosh \left(\frac{q_{1}-q_{2}}{2 k}\right)} \cdot \frac{1}{\left(2 \cosh \frac{q_{2}}{2}\right)^{1 / 2}},
$$

is understood as the density matrix of this Fermi gas system. Since the sum over permutations in (2.3) can be also written as a sum over conjugacy classes of the permutation group, it is convenient to consider the grand partition function

$$
\Xi(z)=1+\sum_{N=1}^{\infty} Z(N) z^{N}
$$

where $z=e^{\mu}$ is the fugacity and $\mu$ is the chemical potential. In our analysis, it is important to notice that the grand partition function has the following form

$$
\Xi(z)=\exp \left[-\sum_{n=1}^{\infty} \frac{(-z)^{n}}{n} \operatorname{Tr} \rho^{n}\right]
$$

where $\operatorname{Tr} \rho^{n}$ is defined by

$$
\operatorname{Tr} \rho^{n}=\int_{-\infty}^{\infty} \frac{d q_{1}}{2 \pi k} \cdots \frac{d q_{n}}{2 \pi k} \rho\left(q_{1}, q_{2}\right) \rho\left(q_{2}, q_{3}\right) \cdots \rho\left(q_{n}, q_{1}\right)
$$

It is easy to see that $\Xi(z)$ can be expressed as the Fredholm determinant of the kernel $\rho 1$

$$
\Xi(z)=\operatorname{det}(1+z \rho)
$$

Once the grand partition function is known, one can obtain the partition function by performing the contour integral

$$
Z(N)=\oint \frac{d z}{2 \pi i} \frac{\Xi(z)}{z^{N+1}}
$$

\footnotetext{
${ }^{1} \mathrm{~A}$ similar analysis of the grand partition function of other matrix models has been appeared in $[28,29,30$.
} 
For later analysis, it is convenient to divide the density matrix into two parts. Since the density matrix $\rho\left(q_{1}, q_{2}\right)$ is parity-preserving: $\rho\left(-q_{1},-q_{2}\right)=\rho\left(q_{1}, q_{2}\right)$, we decompose it into the parity even and odd parts

$$
\rho\left(q_{1}, q_{2}\right)=\rho_{+}\left(q_{1}, q_{2}\right)+\rho_{-}\left(q_{1}, q_{2}\right), \quad \rho_{ \pm}\left(q_{1}, q_{2}\right)=\frac{\rho\left(q_{1}, q_{2}\right) \pm \rho\left(q_{1},-q_{2}\right)}{2} .
$$

By definition, $\rho_{ \pm}\left(q_{1},-q_{2}\right)= \pm \rho_{ \pm}\left(q_{1}, q_{2}\right)$, and thus all the matrix elements of the product $\rho_{+} \rho_{-}$ vanish:

$$
\left(\rho_{+} \rho_{-}\right)\left(q_{1}, q_{2}\right)=\int_{-\infty}^{\infty} \frac{d q}{2 \pi} \rho_{+}\left(q_{1}, q\right) \rho_{-}\left(q, q_{2}\right)=0 .
$$

This means that $\operatorname{Tr} \rho^{n}$ splits into two parts,

$$
\operatorname{Tr} \rho^{n}=\operatorname{Tr} \rho_{+}^{n}+\operatorname{Tr} \rho_{-}^{n} .
$$

and the grand partition function is factorized into

$$
\Xi(z)=\Xi_{+}(z) \Xi_{-}(z)
$$

where

$$
\Xi_{+}(z)=\operatorname{det}\left(1+z \rho_{+}\right), \quad \Xi_{-}(z)=\operatorname{det}\left(1+z \rho_{-}\right) .
$$

As we will see later, such a decomposition of the density matrix plays an important role in the analysis of the grand partition function.

\subsection{Method to compute the grand partition function}

Now let us consider the computation of the grand partition function. As can be seen from (2.6), we can compute the grand partition function if we know $\operatorname{Tr} \rho^{n}$. To compute the partition function $Z(N)$, in particular, we need $\operatorname{Tr} \rho^{n}(1 \leq n \leq N)$. However it is technically difficult to directly compute $\operatorname{Tr} \rho^{n}$ (or the matrix element $\rho^{n}\left(q_{1}, q_{2}\right)$ ) because it is given by the multivariable integral as in (2.7) (or (1.2)). To overcome this difficulty, we here use the technique proposed in [24], which enables us to compute $\rho^{n}\left(q_{1}, q_{2}\right)$ from a series of functions of one variable. This technique can be applicable to a wide class of the kernels with the form

$$
K\left(q_{1}, q_{2}\right)=\frac{E\left(q_{1}\right) E\left(q_{2}\right)}{M\left(q_{1}\right)+M\left(q_{2}\right)} .
$$

Here we apply it to the kernels $\rho_{ \pm}$. Below we concentrate our attention to $k=1$ for simplicity. It is straightforward to generalize the method here to general $k \geq 2$. 
The important point is that two matrices $\rho_{ \pm}\left(q_{1}, q_{2}\right)$ have the following form 2

$$
\rho_{ \pm}\left(q_{1}, q_{2}\right)=\frac{E_{ \pm}\left(q_{1}\right) E_{ \pm}\left(q_{2}\right)}{\cosh q_{1}+\cosh q_{2}}
$$

where

$$
E_{+}(q)=\frac{\cosh \frac{q}{2}}{\sqrt{2 \cosh \frac{q}{2}}}, \quad E_{-}(q)=\frac{\sinh \frac{q}{2}}{\sqrt{2 \cosh \frac{q}{2}}}
$$

We can apply Lemma 1 in [24] to (2.16). It is shown that (the generating functions of) the density matrices are given by

$$
\begin{aligned}
& {\left[\frac{z \rho_{ \pm}}{1-z^{2} \rho_{ \pm}^{2}}\right]\left(q_{1}, q_{2}\right)=\frac{Q_{ \pm}\left(q_{1}\right) Q_{ \pm}\left(q_{2}\right)-P_{ \pm}\left(q_{1}\right) P_{ \pm}\left(q_{2}\right)}{\cosh q_{1}+\cosh q_{2}}} \\
& {\left[\frac{z^{2} \rho_{ \pm}^{2}}{1-z^{2} \rho_{ \pm}^{2}}\right]\left(q_{1}, q_{2}\right)=\frac{Q_{ \pm}\left(q_{1}\right) P_{ \pm}\left(q_{2}\right)-P_{ \pm}\left(q_{1}\right) Q_{ \pm}\left(q_{2}\right)}{\cosh q_{1}-\cosh q_{2}}}
\end{aligned}
$$

where

$$
P_{ \pm}(q)=\int \frac{d q^{\prime}}{2 \pi}\left[\frac{z \rho_{ \pm}}{1-z^{2} \rho_{ \pm}^{2}}\right]\left(q, q^{\prime}\right) \sqrt{z} E_{ \pm}\left(q^{\prime}\right), \quad Q_{ \pm}(q)=\int \frac{d q^{\prime}}{2 \pi}\left[\frac{1}{1-z^{2} \rho_{ \pm}^{2}}\right]\left(q, q^{\prime}\right) \sqrt{z} E_{ \pm}\left(q^{\prime}\right) .
$$

If we expand $P_{ \pm}(q)$ and $Q_{ \pm}(q)$ as

$$
P_{ \pm}(q)=\sqrt{z} E_{ \pm}(q) \sum_{k=0}^{\infty} z^{2 k+1} \phi_{ \pm}^{2 k+1}(q), \quad Q_{ \pm}(q)=\sqrt{z} E_{ \pm}(q) \sum_{k=0}^{\infty} z^{2 k} \phi_{ \pm}^{2 k}(q)
$$

where

$$
\phi_{ \pm}^{k}(q)=\frac{1}{E_{ \pm}(q)} \int \frac{d q^{\prime}}{2 \pi} \rho_{ \pm}^{k}\left(q, q^{\prime}\right) E_{ \pm}\left(q^{\prime}\right)
$$

then (2.18) and (2.19) indicate that the density matrices are given by

$$
\begin{aligned}
\rho_{ \pm}^{2 n+1}\left(q_{1}, q_{2}\right) & =\frac{E_{ \pm}\left(q_{1}\right) E_{ \pm}\left(q_{2}\right)}{\cosh q_{1}+\cosh q_{2}} \sum_{k=0}^{2 n}(-1)^{k} \phi_{ \pm}^{k}\left(q_{1}\right) \phi_{ \pm}^{2 n-k}\left(q_{2}\right) \\
\rho_{ \pm}^{2 n}\left(q_{1}, q_{2}\right) & =\frac{E_{ \pm}\left(q_{1}\right) E_{ \pm}\left(q_{2}\right)}{\cosh q_{1}-\cosh q_{2}} \sum_{k=0}^{2 n-1}(-1)^{k} \phi_{ \pm}^{k}\left(q_{1}\right) \phi_{ \pm}^{2 n-1-k}\left(q_{2}\right)
\end{aligned}
$$

\footnotetext{
${ }^{2}$ Note that

$$
\rho\left(q_{1}, q_{2}\right)=\frac{e^{\frac{1}{2} q_{1}}}{\sqrt{2 \cosh \frac{q_{1}}{2}}} \frac{1}{e^{q_{1}}+e^{q_{2}}} \frac{e^{\frac{1}{2} q_{2}}}{\sqrt{2 \cosh \frac{q_{2}}{2}}}=\frac{e^{-\frac{1}{2} q_{1}}}{\sqrt{2 \cosh \frac{q_{1}}{2}}} \frac{1}{e^{-q_{1}}+e^{-q_{2}}} \frac{e^{-\frac{1}{2} q_{2}}}{\sqrt{2 \cosh \frac{q_{2}}{2}}}
$$
}

itself is also of this class. 
These are the key relations in our analysis later. These results show that the matrix elements $\rho_{ \pm}^{n}\left(q_{1}, q_{2}\right)$ are determined algebraically by the functions $\phi_{ \pm}^{k}(q)(k=0, \ldots, n-1)$ of one variable respectively. Therefore our problem reduces to finding $\phi_{ \pm}^{k}(q)$, which is much simpler than the original one of finding $\rho_{ \pm}^{n}\left(q_{1}, q_{2}\right)$ themselves.

For the determination of $\phi_{ \pm}^{k}(q)$, as discussed in Appendix $\mathrm{A}$, we can show that $\phi_{ \pm}^{k}(q)$ satisfy the following recurrence relations

$$
\phi_{+}^{k}(q)=c_{q} \int_{-\infty}^{\infty} \frac{d q^{\prime}}{2 \pi} c_{q-q^{\prime}} \phi_{+}^{k-1}\left(q^{\prime}\right), \quad \phi_{-}^{k}(q)=c_{q} \int_{-\infty}^{\infty} \frac{d q^{\prime}}{2 \pi} c_{q-q^{\prime}} t_{q^{\prime}}^{2} \phi_{-}^{k-1}\left(q^{\prime}\right) .
$$

Throughout this paper, we often use the notations

$$
c_{q}=\frac{1}{2 \cosh \frac{q}{2}}, \quad s_{q}=\frac{1}{2 \sinh \frac{q}{2}}, \quad t_{q}=\tanh \frac{q}{2} .
$$

From (2.25) with the initial condition $\phi_{ \pm}^{0}(q)=1$, one can recursively fix $\phi_{ \pm}^{k}(q)$. In Section 4, we will indeed determine the first few $\phi_{ \pm}^{k}(q)$ by using these recurrence relations.

Let us summarize our general prescription to compute the grand partition function. We first determine $\phi_{ \pm}^{k}(q)$ by using (2.25). Then from (2.23) and (2.24), we find the matrix elements $\rho_{ \pm}^{n}\left(q_{1}, q_{2}\right)$. Computing $\operatorname{Tr} \rho_{ \pm}^{n}$ from such expressions, we obtain the grand partition function through (2.6) and (2.12).

\subsection{Eigenvalue problem and Hankel matrix representation}

In the previous subsection, we considered $\operatorname{Tr} \rho^{n}$ to know the grand partition function. There is another approach to analyze it. The grand partition function can be expressed in terms of the eigenvalues of the density matrix $\rho$,

$$
\Xi(z)=\operatorname{det}(1+z \rho)=\prod_{n=0}^{\infty}\left(1+z \lambda_{n}\right),
$$

where $\lambda_{n}$ is the $n$-th eigenvalue of $\rho$ with decreasing order $\lambda_{0} \geq \lambda_{1} \geq \cdots$. Thus if we can solve the eigenvalue problem of $\rho$, we obtain $\Xi(z)$. The eigenvalue equation is given by the homogeneous Fredholm integral equation of the second kind

$$
\int_{-\infty}^{\infty} \frac{d q^{\prime}}{2 \pi} \rho\left(q, q^{\prime}\right) \Psi\left(q^{\prime}\right)=\lambda \Psi(q)
$$

It is, of course, hard to solve this equation, but we can rewrite it as the eigenvalue equation for an infinite dimensional Hankel matrix $M$ whose matrix elements are given by

$$
M_{n m}=\frac{1}{8 \pi k} \int_{-\infty}^{\infty} d q \frac{\tanh ^{n+m}\left(\frac{q}{2 k}\right)}{\cosh \left(\frac{q}{2}\right) \cosh ^{2}\left(\frac{q}{2 k}\right)}=\frac{1}{4 \pi} \int_{-1}^{1} d t \frac{t^{n+m}}{T_{k}\left(1 / \sqrt{1-t^{2}}\right)},
$$


where $T_{k}(x)$ is the $k$-th Chebyshev polynomial of the first kind. This representation is useful in our later analysis.

To rewrite the eigenvalue equation, let us first expand the kernel (2.4) into

$$
\rho\left(q_{1}, q_{2}\right)=\sum_{n=0}^{\infty} \rho_{n}\left(q_{1}\right) \rho_{n}\left(q_{2}\right)
$$

where

$$
\rho_{n}(q)=\frac{\tanh ^{n}\left(\frac{q}{2 k}\right)}{2 \sqrt{k \cosh \left(\frac{q}{2}\right)} \cosh \left(\frac{q}{2 k}\right)} .
$$

Since $\rho_{n}(-q)=(-1)^{n} \rho_{n}(q)$, the decomposition (2.10) indicates that $\rho_{ \pm}$are respectively expressed as

$$
\rho_{+}\left(q_{1}, q_{2}\right)=\sum_{n=0}^{\infty} \rho_{2 n}\left(q_{1}\right) \rho_{2 n}\left(q_{2}\right), \quad \rho_{-}\left(q_{1}, q_{2}\right)=\sum_{n=0}^{\infty} \rho_{2 n+1}\left(q_{1}\right) \rho_{2 n+1}\left(q_{2}\right)
$$

Plugging (2.30) into (2.28), we obtain

$$
\sum_{n=0}^{\infty} v_{n} \rho_{n}(q)=\lambda \Psi(q)
$$

where

$$
v_{n}=\int_{-\infty}^{\infty} \frac{d q}{2 \pi} \rho_{n}(q) \Psi(q) .
$$

Substituting (2.33) back to (2.34), we find

$$
\sum_{m=0}^{\infty} M_{n m} v_{m}=\lambda v_{n}
$$

where the matrix elements of $M$ are given by (2.29). The equation (2.35) formally shows that all the eigenvalues of $\rho$ are also the eigenvalues of the infinite dimensional matrix $M$.

The matrix elements $M_{n m}$ depend only on $n+m$, and such matrices are called Hankel matrices. For the present case, it is obvious that $M_{n m}=0$ if $n+m$ is odd. Namely, this Hankel matrix $M$ has the following form

$$
M=\left(\begin{array}{ccccccc}
m_{0} & 0 & m_{1} & 0 & m_{2} & 0 & \cdots \\
0 & m_{1} & 0 & m_{2} & 0 & m_{3} & \\
m_{1} & 0 & m_{2} & 0 & m_{3} & 0 & \\
0 & m_{2} & 0 & m_{3} & 0 & m_{4} & \\
m_{2} & 0 & m_{3} & 0 & m_{4} & 0 & \\
\vdots & & & & & & \ddots
\end{array}\right) .
$$


We shall call such a matrix a parity-preserving Hankel matrix. As can be seen easily, the parity-preserving Hankel matrix can be decomposed into two blocks of Hankel matrices with opposite parity

$$
M_{+}=\left(\begin{array}{cccc}
m_{0} & m_{1} & m_{2} & \cdots \\
m_{1} & m_{2} & m_{3} & \\
m_{2} & m_{3} & m_{4} & \\
\vdots & & & \ddots
\end{array}\right), \quad M_{-}=\left(\begin{array}{cccc}
m_{1} & m_{2} & m_{3} & \cdots \\
m_{2} & m_{3} & m_{4} & \\
m_{3} & m_{4} & m_{5} & \\
\vdots & & & \ddots
\end{array}\right) .
$$

These two Hankel matrices are related by

$$
M_{-}=M_{+} T_{-}=T_{+} M_{+},
$$

with the shift matrix $\left(T_{ \pm}\right)_{m n}=\delta_{m \pm 1, n}$. The eigen-spaces of $M$ also decompose into the direct product of the eigen-spaces of $M_{ \pm}$.

For $k=1, M_{n m}$ has the following very simple form $(n+m$ : even $)$

$$
M_{n m}^{(k=1)}=\frac{C_{\frac{n+m}{2}}}{2^{n+m+3}},
$$

where $C_{n}$ is the Catalan number

$$
C_{n}=\frac{(2 n) !}{(n+1) ! n !}
$$

For $k=2$, we find $(n+m$ : even)

$$
M_{n m}^{(k=2)}=\frac{1}{8 \pi}\left[-\frac{2}{n+m+1}+\psi\left(\frac{n+m+3}{4}\right)-\psi\left(\frac{n+m+1}{4}\right)\right],
$$

where $\psi(x)=\Gamma^{\prime}(x) / \Gamma(x)$ is the digamma function.

After seen the simple Hankel matrix representation, before closing this subsection, now let us express various quantities appearing in the previous subsection in terms of this Hankel matrix $M$. Since the matrix $M$ and the density matrix $\rho$ share the same set of eigenvalues assuming a certain regularity condition, it is obvious that $\operatorname{Tr} M^{n}$ is equal to $\operatorname{Tr} \rho^{n}$ for any $n$, thus the grand partition function is given by

$$
\Xi(z)=\operatorname{det}(1+z M)
$$

Repeating the same argument above, one finds that the eigenvalue problems for $\rho_{ \pm}$are mapped to those for the Hankel matrices $M_{ \pm}$, respectively. Let $\lambda_{ \pm, n}(n=0,1,2, \ldots)$ be the $n$-th 
eigenvalue of the density matrix $\rho_{ \pm}$. Then, we find

$$
\begin{aligned}
& \Xi_{+}(z)=\prod_{n=0}^{\infty}\left(1+z \lambda_{+, n}\right)=\operatorname{det}\left(1+z M_{+}\right), \\
& \Xi_{-}(z)=\prod_{n=0}^{\infty}\left(1+z \lambda_{-, n}\right)=\operatorname{det}\left(1+z M_{-}\right) .
\end{aligned}
$$

We observe from the numerical evaluations (see Section 5.3) that the eigenvalues of $\rho_{ \pm}$satisfy the inequality

$$
\lambda_{+, 0}>\lambda_{-, 0}>\lambda_{+, 1}>\lambda_{-, 1}>\lambda_{+, 2}>\lambda_{-, 2}>\cdots,
$$

which means the following relations

$$
\lambda_{+, n}=\lambda_{2 n}, \quad \lambda_{-, n}=\lambda_{2 n+1} .
$$

Also, $\phi_{+}^{k}(q)$ and its Fourier transformation $\tilde{\phi}_{+}^{k}(p)$

$$
\tilde{\phi}_{+}^{k}(p)=\int \frac{d q}{2 \pi} e^{-\frac{i}{2 \pi} p q} \phi_{+}^{k}(q)
$$

can be expressed in terms of $M$ as

$$
\frac{1}{4} c_{q}^{-2} \phi_{+}^{k}(q)=\sum_{m, n=0}^{\infty} t_{q}^{2 n}\left(M_{+}^{k}\right)_{n m}, \quad 2 c_{q}^{-1} \tilde{\phi}_{+}^{k}(q)=\sum_{n=0}^{\infty} t_{q}^{2 n}\left(M_{+}^{k-1}\right)_{n 0} .
$$

where $t_{q}=\tanh (q / 2)$. The derivation of these representations will be given in Appendix B,

\subsection{Representation in Chebyshev basis}

In the previous subsection, we have found an interesting Hankel matrix representation for the density matrix $\rho\left(q_{1}, q_{2}\right)$. It is natural to wonder whether there is an orthonormal basis in which the density matrix $\rho\left(q_{1}, q_{2}\right)$ is represented by this Hankel matrix. We will show in this subsection that the Hankel matrix $M$ is closely related to the density matrix in a basis constructed by the Chebyshev polynomials.

Motivated by the expansion (2.30), let us introduce the following function

$$
\langle q \mid n\rangle=\frac{\sqrt{2} U_{n}\left(\tanh \left(\frac{q}{2}\right)\right)}{\cosh ^{3 / 2}\left(\frac{q}{2}\right)}
$$

where $U_{n}(x)$ is the Chebyshev polynomial of the second kind and its appearance originates from the orthonomality of the basis $\{|n\rangle\}_{n=0}^{\infty}$,

$$
\langle m \mid n\rangle=\int_{-\infty}^{\infty} \frac{d q}{2 \pi} \frac{\sqrt{2} U_{m}\left(\tanh \left(\frac{q}{2}\right)\right)}{\cosh ^{3 / 2}\left(\frac{q}{2}\right)} \frac{\sqrt{2} U_{n}\left(\tanh \left(\frac{q}{2}\right)\right)}{\cosh ^{3 / 2}\left(\frac{q}{2}\right)}=\delta_{m n} .
$$


Then, the density matrix in this basis, $M_{n_{1} n_{2}}^{\rho}=\left\langle n_{1}|\rho| n_{2}\right\rangle$, is given by

$$
M_{n_{1} n_{2}}^{\rho}=\int \frac{d q_{1}}{2 \pi} \frac{d q_{2}}{2 \pi}\left\langle n_{1} \mid q_{1}\right\rangle\left\langle q_{1}|\rho| q_{2}\right\rangle\left\langle q_{2} \mid n_{2}\right\rangle=\sum_{n=0}^{\infty} \int \frac{d q_{1}}{2 \pi}\left\langle q_{1} \mid n_{1}\right\rangle \rho_{n}\left(q_{1}\right) \int \frac{d q_{2}}{2 \pi}\left\langle q_{2} \mid n_{2}\right\rangle \rho_{n}\left(q_{2}\right) .
$$

where we have used the expansion (2.30). Let $B$ be the matrix for the basis change from Chebyshev polynomials to monomials

$$
t^{n}=\sum_{m=0}^{\infty} B_{n m} U_{m}(t)
$$

The explicit form of the non-vanishing matrix element $B_{n m}$ is given by

$$
B_{n m}=\frac{1}{2^{n}} \frac{m+1}{n+1}\left(\begin{array}{c}
n+1 \\
(n-m) / 2
\end{array}\right)
$$

when $n+m$ is even and $n \geq m$. Since one can check

$$
\int \frac{d q_{1}}{2 \pi}\left\langle q_{1} \mid n_{1}\right\rangle \rho_{n}\left(q_{1}\right)=\sum_{m} B_{n m} \int \frac{d q_{1}}{2 \pi} \frac{U_{n_{1}}\left(\tanh \left(\frac{q_{1}}{2}\right)\right) U_{m}\left(\tanh \left(\frac{q_{1}}{2}\right)\right)}{\sqrt{2} \cosh ^{3}\left(\frac{q_{1}}{2}\right)}=\frac{1}{2 \sqrt{2}} B_{n n_{1}},
$$

the matrix element $M_{n_{1} n_{2}}^{\rho}$ is given by

$$
M_{n_{1} n_{2}}^{\rho}=\frac{1}{8} \sum_{n=0}^{\infty} B_{n n_{1}} B_{n n_{2}}=\frac{1}{8}\left(B^{T} B\right)_{n_{1} n_{2}} .
$$

On the other hand, the Hankel matrix $M$ for $k=1$ is written as

$$
\begin{aligned}
M_{n_{1} n_{2}} & =\frac{1}{8 \pi} \int d q \frac{\tanh ^{n_{1}+n_{2}}\left(\frac{q}{2}\right)}{\cosh ^{3}\left(\frac{q}{2}\right)}=\frac{1}{8 \pi} \sum_{m_{1}, m_{2}} B_{n_{1} m_{1}} B_{n_{2} m_{2}} \int d q \frac{U_{m_{1}}\left(\tanh \left(\frac{q}{2}\right)\right) U_{m_{2}}\left(\tanh \left(\frac{q}{2}\right)\right)}{\cosh ^{3}\left(\frac{q}{2}\right)} \\
& =\frac{1}{8}\left(B B^{T}\right)_{n_{1} n_{2} .} .
\end{aligned}
$$

In summary, the Hankel matrix $M$ and the density matrix $M^{\rho}$ in the Chebyshev basis are written as

$$
M=\frac{1}{8} B B^{T}, \quad M^{\rho}=\frac{1}{8} B^{T} B
$$

These two matrices are isospectral, i.e., they have the same set of eigenvalues (assuming a certain regularity condition)

$$
M v=\lambda v \quad \Leftrightarrow \quad M^{\rho} v^{\prime}=\lambda v^{\prime}, \quad v^{\prime}=B^{T} v .
$$

Note that $M^{\rho}$ is not a Hankel matrix while $M$ is so. 


\section{Algebraic treatment}

Here we consider an algebraic treatment of our Fermi gas system. Note that the matrix element in the position representation (2.4)

$$
\rho\left(q_{1}, q_{2}\right)=\left\langle q_{1}|\hat{\rho}| q_{2}\right\rangle
$$

comes from a one-dimensional quantum mechanical system with the operator $\hat{\rho}$ expressed in terms of the coordinate $\hat{q}$ and the momentum $\hat{p}$ as

$$
\hat{\rho}=\sqrt{c_{\hat{q}}} c_{\hat{p}} \sqrt{c_{\hat{q}}}
$$

Below we focus on the $k=1$ case. Let us summarize our convention for the phase space used in this paper, which is especially clean for $k=1$. The commutation relation of $\hat{q}$ and $\hat{p}$ is given by

$$
[\hat{q}, \hat{p}]=2 \pi i
$$

The normalization of the bases $|q\rangle$ and $|p\rangle$ is as follows:

$$
\left\langle q \mid q^{\prime}\right\rangle=2 \pi \delta\left(q-q^{\prime}\right), \quad\left\langle p \mid p^{\prime}\right\rangle=2 \pi \delta\left(p-p^{\prime}\right), \quad\langle q \mid p\rangle=e^{\frac{i}{2 \pi} p q}
$$

Therefore the projectors are normalized as

$$
1=\int_{-\infty}^{\infty} \frac{d q}{2 \pi}|q\rangle\left\langle q\left|=\int_{-\infty}^{\infty} \frac{d p}{2 \pi}\right| p\right\rangle\langle p|
$$

Hereafter, we suppress the hats on operators for simplicity. Note that the matrix elements of $c_{p}$ and $s_{p}$ in the position representation are

$$
\left\langle q_{1}\left|c_{p}\right| q_{2}\right\rangle=c_{q_{1}-q_{2}}, \quad\left\langle q_{1}\left|s_{p}\right| q_{2}\right\rangle=\frac{i}{2} \tanh \left(\frac{q_{1}-q_{2}}{2}\right) .
$$

Using the bra-ket notation, the matrix elements of $\rho^{n}$ are given by

$$
\rho^{n}\left(q_{1}, q_{2}\right)=\left\langle q_{1}\left|\left(\sqrt{c_{q}} c_{p} \sqrt{c_{q}}\right)^{n}\right| q_{2}\right\rangle=\sqrt{\frac{c_{q_{1}}}{c_{q_{2}}}}\left\langle q_{1}\left|\left(c_{p} c_{q}\right)^{n}\right| q_{2}\right\rangle .
$$

Since $\operatorname{Tr} \rho^{n}=\operatorname{Tr}\left[\left(c_{p} c_{q}\right)^{n}\right]=\operatorname{Tr}\left[\left(c_{q} c_{p}\right)^{n}\right]$ from this equation, the grand partition function is also written as

$$
\Xi(z)=\operatorname{det}\left(1+z c_{p} c_{q}\right)=\operatorname{det}\left(1+z c_{q} c_{p}\right)
$$




\subsection{Anomalous terms in commutation relations}

As explained around (1.2), the difficulty in computing $\operatorname{Tr} \rho^{n}$ consists in the multi-dimensional integral. In the operator representation, it can also be stated as the difficulty in the integration over the multi-dimensional phase space. Therefore, we would like to reduce the dimension.

If we know the commutation relation between $c_{p}$ and $c_{q}$, we can reduce the dimension of the phase space involved. As is seen here, one should be careful to derive the commutation relation. From the operator identity

$$
2 \cosh \frac{q}{2} \cdot 2 \cosh \frac{p}{2}=i \cdot 2 \sinh \frac{p}{2} \cdot 2 \sinh \frac{q}{2},
$$

one would naively expect

$$
\frac{1}{2 \cosh \frac{p}{2}} \cdot \frac{1}{2 \cosh \frac{q}{2}} \stackrel{?}{=}-i \cdot \frac{1}{2 \sinh \frac{q}{2}} \cdot \frac{1}{2 \sinh \frac{p}{2}} .
$$

However this is not the case. There is an anomalous term in this commutation relation. To see this, let us consider the matrix element of the difference between both sides of (3.10)

$$
\left\langle q_{1}\left|\frac{1}{2 \cosh \frac{p}{2}} \cdot \frac{1}{2 \cosh \frac{q}{2}}\right| q_{2}\right\rangle=-i\left\langle q_{1}\left|\frac{1}{2 \sinh \frac{q}{2}} \cdot \frac{1}{2 \sinh \frac{p}{2}}\right| q_{2}\right\rangle+\frac{1}{4 \sinh \frac{q_{1}}{2}} \tanh \frac{q_{2}}{2} .
$$

If we introduce a projection operator $|p=0\rangle\left\langle q=0|=:| 0_{p}\right\rangle\left\langle 0_{q}\right|$ satisfying $\left(\left|0_{p}\right\rangle\left\langle 0_{q}\right|\right)^{2}=\left|0_{p}\right\rangle\left\langle 0_{q}\right|$, we can express the relation as

$$
c_{p} c_{q}=-i s_{q} \Pi s_{p}, \quad \Pi=1-\left|0_{p}\right\rangle\left\langle 0_{q}\right|,
$$

at the operator level. Similarly, we find

$$
c_{q} c_{p}=i s_{p} \Pi^{\dagger} s_{q}, \quad \Pi^{\dagger}=1-\left|0_{q}\right\rangle\left\langle 0_{p}\right| .
$$

Using the commutation relations (3.12) and (3.13), we can calculate $\operatorname{Tr} \rho^{n}$ correctly. Let us see the computation of $\operatorname{Tr} \rho^{2}$ as a simple example:

$$
\operatorname{Tr} \rho^{2}=\operatorname{Tr}\left(c_{p} c_{q} c_{p} c_{q}\right)=-i \operatorname{Tr}\left(s_{q} \Pi s_{p} c_{p} c_{q}\right)=-i \operatorname{Tr}\left(s_{2 q} \Pi s_{2 p}\right)
$$

where we have used $s_{q} c_{q}=s_{2 q}$. Since $\Pi=1-\left|0_{p}\right\rangle\left\langle 0_{q}\right|$, (3.14) is further rewritten as

$$
\operatorname{Tr} \rho^{2}=-i \int \frac{d q d p}{(2 \pi)^{2}} s_{2 q} s_{2 p}+i \int \frac{d q}{2 \pi}\left\langle q\left|s_{2 q}\right| 0_{p}\right\rangle\left\langle 0_{q}\left|s_{2 p}\right| q\right\rangle
$$

The first term vanishes because the integrand is an odd function. The second term is evaluated as

$$
i \int \frac{d q}{2 \pi}\left\langle q\left|s_{2 q}\right| 0_{p}\right\rangle\left\langle 0_{q}\left|s_{2 p}\right| q\right\rangle=\frac{1}{4} \int \frac{d q}{2 \pi} c_{q} c_{q / 2}^{2}
$$


where $\left\langle q\left|s_{2 q}\right| 0_{p}\right\rangle=s_{2 q}$ and $\left\langle 0_{q}\left|s_{2 p}\right| q\right\rangle=-(i / 4) \tanh (q / 4)$. Therefore we obtain

$$
\operatorname{Tr} \rho^{2}=\frac{\pi-2}{16 \pi} \text {. }
$$

One can easily check that the same result is reproduced by a brute force integration using (2.7). In the above computation, it is obvious that the anomalous term in the commutation relation is crucial to obtain the correct answer. In principle, we can compute $\operatorname{Tr} \rho^{n}$ by using the relations (3.12) and (3.13) repeatedly instead of doing multi-integral. In practice, however, the computation becomes very complicated as $n$ grows. One advantage to consider the algebraic treatment is to give us some non-trivial implications for the grand partition function as we see in the next subsection.

\subsection{Implications for the grand partition function}

In the previous subsection, we originally intended to reduce the dimensions of the phase space integration by commuting the coordinate $q$ and the momentum $p$. Though this was not successful because of an anomaly term, the anomaly term is controlable and gives us an algebraic method to compute $\operatorname{Tr} \rho^{n}$. In this subsection, we would like to discuss a more important implication.

Using (3.13) and (3.12), it is not difficult to show

$$
\operatorname{Tr}\left(c_{q} c_{p} \Pi\right)^{n}=i^{n} \operatorname{Tr}\left(s_{p} \Pi^{\dagger} s_{q} \Pi\right)^{n}=i^{n} \operatorname{Tr}\left(\Pi^{\dagger} s_{q} \Pi s_{p}\right)^{n}=i^{2 n} \operatorname{Tr}\left(\Pi^{\dagger} c_{p} c_{q}\right)^{n} .
$$

Since $\operatorname{Tr}\left(c_{q} c_{p} \Pi\right)^{n}$ is real, this should be equal to its conjugate $\operatorname{Tr}\left(\Pi^{\dagger} c_{p} c_{q}\right)^{n}$. The observation (3.18) shows that, if the power $n$ is odd, we have $\operatorname{Tr}\left(c_{q} c_{p} \Pi\right)^{n}=0$ which implies that, by expanding $\Pi=1-\left|0_{p}\right\rangle\left\langle 0_{q}\right|, \operatorname{Tr} \rho^{n}=\operatorname{Tr}\left(c_{q} c_{p}\right)^{n}$ can be expressed in terms of a certain combination of $\left\langle 0_{q}\left|\left(c_{q} c_{p}\right)^{m}\right| 0_{p}\right\rangle$. The easiest way to incorporate the combinatorics correctly is as follows.

Let us consider the determinant $\Xi_{1}(z)=\operatorname{det}\left(1+z c_{p} \Pi c_{q}\right)$. This determinant is rewritten as

$$
\Xi_{1}(z)=\operatorname{det}\left(1+z c_{p} c_{q}-\frac{z}{4}\left|0_{p}\right\rangle\left\langle 0_{q}\right|\right)
$$

where we have used $c_{q}\left|0_{q}\right\rangle=(1 / 2)\left|0_{q}\right\rangle$. This determinant is formally written as

$$
\Xi_{1}(z)=\operatorname{det}\left(1+z c_{p} c_{q}\right) \operatorname{det}\left(1-\frac{z}{4} \frac{1}{1+z c_{p} c_{q}}\left|0_{p}\right\rangle\left\langle 0_{q}\right|\right) .
$$

The first factor is just the grand partition function $\Xi(z)$, and (the logarithm of) the second factor is evaluated as

$$
\log \operatorname{det}\left(1-\frac{z}{4} \frac{1}{1+z c_{p} c_{q}}\left|0_{p}\right\rangle\left\langle 0_{q}\right|\right)=-\operatorname{Tr} \sum_{n=1}^{\infty} \frac{1}{n}\left(\frac{z}{4} \frac{1}{1+z c_{p} c_{q}}\left|0_{p}\right\rangle\left\langle 0_{q}\right|\right)^{n} .
$$


Due to the insertion of the projection operator, the last equation is drastically simplified

$$
-\sum_{n=1}^{\infty} \frac{1}{n}\left(\frac{z}{4}\right)^{n}\left(\left\langle 0_{q}\left|\frac{1}{1+z c_{p} c_{q}}\right| 0_{p}\right\rangle\right)^{n}=\log \left(1-\frac{z}{4}\left\langle 0_{q}\left|\frac{1}{1+z c_{p} c_{q}}\right| 0_{p}\right\rangle\right) .
$$

Therefore the relation (3.20) becomes

$$
\Xi_{1}(z)=\Xi(z) G(-z)
$$

where we have introduced the new function $G(z)$ by

$$
G(z)=1+\frac{z}{4}\left\langle 0_{q}\left|\frac{1}{1-z c_{p} c_{q}}\right| 0_{p}\right\rangle=\left\langle 0_{q}\left|\frac{1}{1-z c_{q} c_{p}}\right| 0_{p}\right\rangle .
$$

Let us show that $\Xi_{1}(z)$ is invariant under $z \rightarrow-z$. Using the commutation relations (3.13) and (3.12), we have

$$
\Xi_{1}(z)=\operatorname{det}\left(1+z \Pi c_{q} c_{p}\right)=\operatorname{det}\left(1+z \Pi i s_{p} \Pi^{\dagger} s_{q}\right)=\operatorname{det}\left(1-z c_{p} c_{q} \Pi^{\dagger}\right) .
$$

Since $\Xi_{1}(z)$ is a real function for $z \in \mathbb{R}$, this implies

$$
\Xi_{1}(z)=\operatorname{det}\left(1-z\left(c_{p} c_{q} \Pi^{\dagger}\right)^{\dagger}\right)=\operatorname{det}\left(1-z \Pi c_{q} c_{p}\right)=\Xi_{1}(-z) .
$$

Plugging (3.23) into (3.26), we find a relation

$$
\frac{\Xi(z)}{\Xi(-z)}=\frac{G(z)}{G(-z)}
$$

Now note that the left hand side is nothing but (the square of) the grand partition function with odd power terms. More explicitly, let us divide $\Xi(z)$ into two parts

$$
\Xi(z)=\Xi_{\text {odd }}(z) \Xi_{\text {even }}(z),
$$

where

$$
\Xi_{\text {odd }}(z)=\exp \left[-\sum_{n=1}^{\infty} \frac{(-z)^{2 n-1}}{2 n-1} \operatorname{Tr} \rho^{2 n-1}\right], \quad \Xi_{\text {even }}(z)=\exp \left[-\sum_{n=1}^{\infty} \frac{(-z)^{2 n}}{2 n} \operatorname{Tr} \rho^{2 n}\right] .
$$

By construction, one easily finds

$$
\Xi_{\text {odd }}(-z)=\frac{1}{\Xi_{\text {odd }}(z)}, \quad \Xi_{\text {even }}(-z)=\Xi_{\text {even }}(z) .
$$

Then, (3.27) shows

$$
\Xi_{\text {odd }}(z)^{2}=\frac{\Xi(z)}{\Xi(-z)}=\frac{G(z)}{G(-z)}
$$


Using this relation, we can compute $\operatorname{Tr} \rho^{2 n+1}$ from $G(z)$. As we will see in the next section, the function $G(z)$ also has an important meaning for the even power terms, which connects the two determinants $\Xi_{+}(z)$ and $\Xi_{-}(z)$ defined in the previous subsection.

After introducing the bra-kets such as $\left\langle 0_{p}\right|$, we can simplify various definitions and relations appearing previously. Using the bra-ket notations, the functions $\phi_{+}^{k}(q)$ and $\tilde{\phi}_{+}^{k}(q)$ are expressed as

$$
\phi_{+}^{k}(q)=\left\langle q\left|\left(c_{q} c_{p}\right)^{k}\right| 0_{p}\right\rangle, \quad \tilde{\phi}_{+}^{k}(q)=\left\langle q\left|\left(c_{p} c_{q}\right)^{k}\right| 0_{q}\right\rangle
$$

It is easily shown that $\phi_{+}^{k}(q)$ satisfies the recurrence relation (2.25)

$$
\phi_{+}^{k}(q)=\int \frac{d q^{\prime}}{2 \pi}\left\langle q\left|c_{q} c_{p}\right| q^{\prime}\right\rangle\left\langle q^{\prime}\left|\left(c_{q} c_{p}\right)^{k-1}\right| 0_{p}\right\rangle=c_{q} \int \frac{d q^{\prime}}{2 \pi} c_{q-q^{\prime}} \phi_{+}^{k-1}\left(q^{\prime}\right) .
$$

Similarly, $\tilde{\phi}_{+}^{k}(q)$ also satisfies the recurrence relation

$$
\tilde{\phi}_{+}^{k}(q)=\int \frac{d q^{\prime}}{2 \pi} c_{q-q^{\prime}} c_{q^{\prime}} \tilde{\phi}_{+}^{k-1}\left(q^{\prime}\right)
$$

Finally let us comment on some useful algebraic relations steming from (2.23) and (2.24) by setting one of the coordinate to zero. First, comparing (3.32) with (3.7), one finds the relation between $\rho^{k}$ and $\tilde{\phi}_{+}^{k}$

$$
\rho^{k}(q, 0)=\sqrt{2 c_{q}} \tilde{\phi}_{+}^{k}(q)
$$

Since $\rho_{-}^{k}(q, 0)=0$ for $k \geq 1, \rho^{k}(q, 0)=\rho_{+}^{k}(q, 0)$ holds. Secondly, by definition of $G(z)$, the expansion of $G(z)$ is given by

$$
G(z)=\sum_{n=0}^{\infty} g_{n} z^{n}, \quad g_{n}=\left\langle 0_{q}\left|\left(c_{q} c_{p}\right)^{n}\right| 0_{p}\right\rangle .
$$

From (3.32) and (3.36), one immediately finds

$$
\phi_{+}^{k}(0)=g_{k} .
$$

Thus, with (3.35) and (3.37), (2.23) and (2.24) imply novel relations between $\phi_{+}^{k}(q)$ and $\tilde{\phi}_{+}^{k}(q)$

$$
\begin{aligned}
\tilde{\phi}_{+}^{2 n+1}(q) & =\frac{1}{4 \cosh \left(\frac{q}{2}\right)} \sum_{k=0}^{2 n}(-1)^{k} \phi_{+}^{k}(q) g_{2 n-k}, \\
\tilde{\phi}_{+}^{2 n}(q) & =\frac{\cosh \left(\frac{q}{2}\right)}{4 \sinh ^{2}\left(\frac{q}{2}\right)} \sum_{k=0}^{2 n-1}(-1)^{k} \phi_{+}^{k}(q) g_{2 n-1-k} .
\end{aligned}
$$

Recall that $\phi_{+}^{k}(q)$ and $\tilde{\phi}_{+}^{k}(q)$ are related through the Fourier transformation originally. 


\section{Exact results}

In this section, we give some exact results for the grand partition function at $k=1$. After the study of the algebraic treatment in the previous section, our strategy to compute $\operatorname{Tr} \rho^{n}$ explained in Section 2 is improved. There are three steps to know $\operatorname{Tr} \rho^{n}$. We first fix $\phi_{ \pm}^{k}(q)$ by using the recurrence relations (2.25). We then compute $\operatorname{Tr} \rho^{n}$ separately for odd $n$ and for even $n$. The computation of $\operatorname{Tr} \rho^{2 n+1}$ reduces to the problem to compute the expansion coefficients $g_{n}$ of $G(z)$, which are fixed by $\phi_{+}^{k}(q)$. For the computation of $\operatorname{Tr} \rho^{2 n}$, on the other hand, we use the formula (2.24). From this prescription, we have determined the exact partition functions of the ABJM theory at $k=1$ up to $N=9$.

\subsection{Exact partition functions}

Let us start by determining $\phi_{ \pm}^{k}(q)$. Since the integral equations (2.25) have the convolution integral, the Fourier transformation is useful to solve (2.25). Let us consider $\phi_{+}^{k}(q)$. The initial condition is $\phi_{+}^{0}(q)=1$. One can trivially find $\phi_{+}^{1}(q)=c_{q} / 2$. Using the Fourier transformation, the convolution between $c_{q}$ and $\phi_{+}^{1}(q)$ can be evaluated as

$$
\int \frac{d q^{\prime}}{2 \pi} c_{q-q^{\prime}} \phi_{+}^{1}\left(q^{\prime}\right)=\frac{1}{2} \int \frac{d p}{2 \pi} e^{\frac{i}{2 \pi} p q} c_{p}^{2}=\frac{1}{4 \pi} q s_{q}
$$

where we have used the formula

$$
c_{p}=\int \frac{d q}{2 \pi} e^{-\frac{i}{2 \pi} p q} c_{q}
$$

Thus we obtain $\phi_{+}^{2}(q)=q s_{2 q} /(4 \pi)$. Iterating this method, we can fix $\phi_{+}^{k}(q)$ recursively. We list the explicit forms of the first seven functions below

$$
\begin{aligned}
\phi_{+}^{0}(q)= & 1, \quad \phi_{+}^{1}(q)=\frac{1}{2} c_{q}, \quad \phi_{+}^{2}(q)=\frac{1}{4 \pi} q s_{2 q}, \quad \phi_{+}^{3}(q)=\frac{1}{16 \pi} c_{q}\left(\pi c_{q}-2 q s_{2 q}\right), \\
\phi_{+}^{4}(q)= & \frac{1}{128 \pi^{2}}\left(4 q^{2} s_{2 q}^{2}+\pi^{2}\left(c_{q}-c_{q}^{2}-c_{q / 2}^{2}\right)\right), \\
\phi_{+}^{5}(q)= & \frac{1}{256 \pi^{2}} c_{q}\left(4 q^{2} s_{2 q}^{2}+4 \pi c_{q}\left(q s_{2 q}+1\right)-\pi^{2}\left(3 c_{q}^{2}+c_{q / 2}^{2}\right)\right), \\
\phi_{+}^{6}(q)= & \frac{1}{384 \pi^{3}} q^{3} s_{q}^{3} c_{q}^{3}+\frac{1}{512 \pi} q s_{q} c_{q}^{3}\left(1+4 c_{q / 2}^{2}\right)-\frac{1}{1536 \pi}\left(16 c_{q}^{3}+2 c_{q}^{2}+c_{q}-c_{q / 2}^{2}\right) \\
& +\frac{1}{24 \pi}\left(q s_{q}-1\right) s_{q}^{2} c_{q}^{3}+\frac{1}{1024} c_{q}^{2}\left(1+2 c_{q}\right) .
\end{aligned}
$$


Similarly $\phi_{-}^{k}(q)$ is also fixed. The explicit forms for $0 \leq k \leq 6$ are given by

$$
\begin{aligned}
\phi_{-}^{0}(q)= & 1, \quad \phi_{-}^{1}(q)=\frac{1}{2} c_{q / 2}^{2}, \quad \phi_{-}^{2}(q)=\frac{1}{4 \pi} c_{q}\left(q s_{q}-\pi c_{q / 2}^{2}\right), \\
\phi_{-}^{3}(q)= & \frac{1}{16 \pi} s_{q}^{2}\left(4-2 q s_{q}\left(1+2 c_{q}\right)-\pi\left(1-2 c_{q}\right)\right) \\
\phi_{-}^{4}(q)= & \frac{1}{32 \pi^{2}} q^{2} s_{2 q}^{2}+\frac{1}{32 \pi} s_{q}^{2}\left(4\left(1-c_{q}\right)-2 q s_{2 q}\left(1+2 c_{q}\right)\right)-\frac{1}{128} c_{q} c_{q / 2}^{2}\left(6 c_{q}+5\right), \\
\phi_{-}^{5}(q)= & \frac{1}{64 \pi^{2}}\left(q^{2} s_{q}^{2} c_{q}^{2} c_{q / 2}^{2}+8 q s_{q}^{3} c_{q}^{2}+4 q s_{q} c_{q}^{2} c_{q / 2}^{2}-2 s_{q}^{2}\right) \\
& +\frac{1}{64 \pi}\left(-4 q s_{q}^{3} c_{q}^{2}-q s_{q} c_{q}^{2} c_{q / 2}^{2}+3 s_{q}^{2}-4 s_{q}^{2} c_{q}\right) \\
& +\frac{1}{256} s_{q / 2}^{2}\left(12 c_{q}^{2} c_{q / 2}^{4}+8 c_{q} c_{q / 2}^{4}+5 c_{q / 2}^{4}-3 c_{q / 2}^{2}\right), \\
\phi_{-}^{6}(q)= & \frac{1}{384 \pi^{3}} q^{3} s_{q}^{3} c_{q}^{3}+\frac{1}{128 \pi^{2}}\left(-q^{2} s_{q}^{2} c_{q}^{3} c_{q / 2}^{2}+8 q s_{q}^{3} c_{q}^{3}+4 q s_{q} c_{q}^{3} s_{q / 2}^{2}+4 q s_{q} c_{q}^{3}\right. \\
& +\frac{1}{1536 \pi}\left(288 s_{q}^{4} c_{q}^{2}-72 s_{q}^{4}+52 q s_{q}^{3} c_{q / 2}^{2}+26 c_{q}^{2} c_{q / 2}^{2}-24 c_{q}^{3}-4 c_{q}^{2}+456 s_{q}^{2} c_{q}^{3}-12 q s_{q}^{3} c_{q}^{2}-15 q s_{q}^{3} c_{q}^{3}+160 s_{q / 2}^{2} c_{q}^{2}-36 s_{q}^{2} c_{q}+20 c_{q}^{2}\right) \\
& +\frac{1}{1024}\left(-32 s_{q}^{2} c_{q}^{3} c_{q / 2}^{2}+224 s_{q}^{2} c_{q}^{3}-54 c_{q}^{3} s_{q / 2}^{2}+72 c_{q}^{3} c_{q / 2}^{2}+12 c_{q}^{2} c_{q / 2}^{2}-6 c_{q}^{3}-c_{q}^{2}\right) .
\end{aligned}
$$

Now we proceed to computing $\operatorname{Tr} \rho^{2 n+1}$. For this purpose, let us start by computing $g_{n}=\left\langle 0_{q}\left|\left(c_{q} c_{p}\right)^{n}\right| 0_{p}\right\rangle$. It is easy to see that $g_{n}$ has the following integral form

$$
g_{n}=\int \frac{d q}{2 \pi}\left\langle 0_{q}\left|\left(c_{q} c_{p}\right)^{m}\right| q\right\rangle\left\langle q\left|\left(c_{q} c_{p}\right)^{n-m}\right| 0_{p}\right\rangle=\int \frac{d q}{2 \pi} \tilde{\phi}_{+}^{m}(q) \phi_{+}^{n-m}(q) .
$$

Note that $g_{n}$ obviously does not depend on the insertion position $m(m=0, \cdots, n)$, thus we can choose it handily. If $m=0$ is chosen, we obtain (3.37) because of $\tilde{\phi}_{+}^{0}(q)=2 \pi \delta(q)$. Using the results (4.3), we find the first fourteen values of $g_{n}$

$$
\begin{aligned}
g_{0}= & 1, \quad g_{1}=\frac{1}{4}, \quad g_{2}=\frac{1}{8 \pi}, \quad g_{3}=\frac{\pi-2}{64 \pi}, \quad g_{4}=\frac{1}{2^{7} \pi^{2}}, \quad g_{5}=\frac{-\pi^{2}+3 \pi+1}{2^{9} \pi^{2}}, \\
g_{6}= & \frac{9 \pi^{3}-28 \pi^{2}+6}{2^{11} \cdot 3^{2} \pi^{3}}, \quad g_{7}=\frac{-45 \pi^{3}+92 \pi^{2}+162 \pi-12}{2^{14} \cdot 3^{2} \pi^{3}}, \\
g_{8}= & \frac{18 \pi^{3}-56 \pi^{2}+3}{2^{15} \cdot 3^{2} \pi^{4}}, \quad g_{9}=\frac{72 \pi^{4}-219 \pi^{3}-74 \pi^{2}+162 \pi+3}{2^{17} \cdot 3^{2} \pi^{4}}, \\
g_{10}= & \frac{-2475 \pi^{5}+5484 \pi^{4}+7650 \pi^{3}-1400 \pi^{2}+30}{2^{19} \cdot 3^{2} \cdot 5^{2} \pi^{5}}, \\
g_{11}= & \frac{4950 \pi^{5}-8684 \pi^{4}-22725 \pi^{3}+1700 \pi^{2}+6075 \pi-30}{2^{22} \cdot 3^{2} \cdot 5^{2} \pi^{5}}, \\
g_{12}= & \frac{2025 \pi^{6}-57150 \pi^{4}+118312 \pi^{4}+132300 \pi^{3}-8400 \pi^{2}+90}{2^{23} \cdot 3^{4} \cdot 5^{2} \pi^{6}}, \\
g_{13}= & \frac{-168075 \pi^{6}+493668 \pi^{5}+273112 \pi^{4}-522450 \pi^{3}-9750 \pi^{2}+65610 \pi+90}{2^{25} \cdot 3^{4} \cdot 5^{2} \pi^{6}} .
\end{aligned}
$$


These lead to the exact value of $\operatorname{Tr} \rho^{2 n+1}$ from (3.31)

$$
\begin{aligned}
\operatorname{Tr} \rho & =\frac{1}{4}, \quad \operatorname{Tr} \rho^{3}=\frac{\pi-3}{16 \pi}, \quad \operatorname{Tr} \rho^{5}=\frac{10-\pi^{2}}{256 \pi^{2}}, \\
\operatorname{Tr} \rho^{7} & =\frac{-27 \pi^{3}+49 \pi^{2}+126 \pi-42}{2^{10} \cdot 3^{2} \pi^{3}}, \quad \operatorname{Tr} \rho^{9}=\frac{5 \pi^{4}-20 \pi^{2}-96 \pi+12}{2^{15} \pi^{4}}, \\
\operatorname{Tr} \rho^{11} & =\frac{4725 \pi^{5}-6303 \pi^{4}-25300 \pi^{3}-12100 \pi^{2}+23100 \pi-660}{2^{17} \cdot 3^{2} \cdot 5^{2} \pi^{5}}, \\
\operatorname{Tr} \rho^{13} & =\frac{-30375 \pi^{6}+10114 \pi^{4}+655200 \pi^{3}+978900 \pi^{2}-561600 \pi+4680}{2^{21} \cdot 3^{4} \cdot 5^{2} \pi^{6}} .
\end{aligned}
$$

Finally let us see the computation of $\operatorname{Tr} \rho^{2 n}$. We have already determined $\phi_{ \pm}^{k}(q)$. Using these results, we can know the matrix elements $\rho_{ \pm}^{k}\left(q_{1}, q_{2}\right)$. The explicit forms of $\rho_{ \pm}^{k}\left(q_{1}, q_{2}\right)$ are given in Appendix C. These matrix elements enable us to compute $\operatorname{Tr} \rho_{ \pm}^{n}$. Here we give the exact values of $\operatorname{Tr} \rho_{ \pm}^{2 n}(1 \leq n \leq 4)$

$$
\begin{aligned}
& \operatorname{Tr} \rho_{+}^{2}=\frac{1}{16 \pi}, \quad \operatorname{Tr} \rho_{+}^{4}=\frac{\pi^{2}-8}{512 \pi^{2}}, \quad \operatorname{Tr} \rho_{+}^{6}=\frac{9 \pi-28}{2^{12} \cdot 3 \pi}, \\
& \operatorname{Tr} \rho_{+}^{8}=\frac{-87 \pi^{2}+192 \pi+256}{2^{17} \cdot 3 \pi^{2}},
\end{aligned}
$$

and

$$
\begin{aligned}
& \operatorname{Tr} \rho_{-}^{2}=\frac{\pi-3}{16 \pi}, \quad \operatorname{Tr} \rho_{-}^{4}=\frac{11 \pi^{2}-32 \pi-8}{512 \pi^{2}}, \quad \operatorname{Tr} \rho_{-}^{6}=\frac{-11 \pi^{2}+4 \pi+96}{2^{12} \pi^{2}}, \\
& \operatorname{Tr} \rho_{-}^{8}=\frac{-1467 \pi^{3}+3520 \pi^{2}+5376 \pi-6144}{2^{17} \cdot 3^{2} \pi^{3}} .
\end{aligned}
$$

Note that $\operatorname{Tr} \rho^{n}$ is computed by using (2.12).

Collecting the above results, the grand partition function is expanded as

$$
\begin{aligned}
\Xi(z)= & 1+\frac{z}{2^{2}}+\frac{z^{2}}{2^{4} \pi}+\frac{\pi-3}{2^{6} \pi} z^{3}+\frac{10-\pi^{2}}{2^{10} \pi^{2}} z^{4}+\frac{-9 \pi^{2}+20 \pi+26}{2^{12} \pi^{2}} z^{5}+\frac{36 \pi^{3}-121 \pi^{2}+78}{2^{14} \cdot 3^{2} \pi^{3}} z^{6} \\
& +\frac{-75 \pi^{3}+193 \pi^{2}+174 \pi-126}{2^{16} \cdot 3 \pi^{3}} z^{7}+\frac{1053 \pi^{4}-2016 \pi^{3}-4148 \pi^{2}+876}{2^{21} \cdot 3^{2} \pi^{4}} z^{8} \\
& +\frac{5517 \pi^{4}-13480 \pi^{3}-15348 \pi^{2}+8880 \pi+4140}{2^{23} \cdot 3^{2} \pi^{4}} z^{9}+\mathcal{O}\left(z^{10}\right) .
\end{aligned}
$$

Let us recall that the coefficient of $z^{N}$ in $\Xi(z)$ is just the partition function $Z(N)$, thus we obtain the exact ABJM partition function $Z(N)$ at $k=1$ up to $N=9$. The partition functions for higher $N$ can also be computed in this way.

\subsection{Novel relation between $G(z)$ and $\Xi_{ \pm}(z)$}

From the results in the previous subsection, we find a novel relation

$$
\log G(z)=\sum_{n=1}^{\infty} \frac{z^{2 n-1}}{2 n-1} \operatorname{Tr} \rho^{2 n-1}+\sum_{n=1}^{\infty} \frac{z^{2 n}}{2 n}\left(\operatorname{Tr} \rho_{+}^{2 n}-\operatorname{Tr} \rho_{-}^{2 n}\right)+\mathcal{O}\left(z^{10}\right),
$$


in the expansion around $z=0$. As we will see below, it is shown that the above relation holds exactly for all $z$, that is,

$$
G(z)=\frac{\operatorname{det}\left(1+z \rho_{-}\right)}{\operatorname{det}\left(1-z \rho_{+}\right)}=\frac{\Xi_{-}(z)}{\Xi_{+}(-z)} .
$$

Before proceeding to the proof, let us comment on a consequence led by the above equality. Using (4.12), the grand partition function is written as

$$
\Xi(z)=\operatorname{det}\left(1-z^{2} \rho_{+}^{2}\right) G(z)
$$

Since the expansion coefficients of $G(z)$ are just given by $\phi_{+}^{k}(0)$ (see (3.37)), the function $G(z)$ is fixed by $\phi_{+}^{k}(0)$. On the other hand, the matrix elements $\rho_{+}^{2 n}\left(q_{1}, q_{2}\right)$ are fixed by $\phi_{+}^{k}(q)$ through (2.24). Therefore $\operatorname{det}\left(1-z^{2} \rho_{+}^{2}\right)$ is also determined by $\phi_{+}^{k}(q)$. We conclude that the total grand partition function is completely fixed by $\phi_{+}^{k}(q)$ (or $\rho_{+}$) only! We do not need any information about $\phi_{-}^{k}(q)$ (or $\rho_{-}$) to know $\Xi(z)$. The novel relation we have found in this section suggests that we have an alternative strategy for computing the grand partition function. Previously we need to compute $\operatorname{Tr} \rho^{n}$. In particular, to compute $\operatorname{Tr} \rho^{2 n}$, we need both of $\operatorname{Tr} \rho_{ \pm}^{2 n}$ and sum up them: $\operatorname{Tr} \rho^{2 n}=\operatorname{Tr} \rho_{+}^{2 n}+\operatorname{Tr} \rho_{-}^{2 n}$. After knowing the relation, the ingredients we need are the coefficients $g_{n}$ of $G(z)$ and $\operatorname{Tr} \rho_{+}^{2 n} \cdot \operatorname{Tr} \rho_{-}^{2 n}$ is automatically fixed by the above relation.

The rest of this subsection is devoted to the proof of (4.12) by using the properties of the parity-preserving Hankel matrix $M$. Using the relation (3.31), one can check that the odd power part of (4.12) holds for all-order in $z$. Thus we concentrate ourselves to the even power part. The statement to be shown is now rephrased as

$$
G(z) G(-z)=\frac{\operatorname{det}\left(1-z^{2} \rho_{-}^{2}\right)}{\operatorname{det}\left(1-z^{2} \rho_{+}^{2}\right)} .
$$

Let us recall that $M$ decomposes into two Hankel matrices $M_{ \pm}$. From (2.38), we find

$$
M_{-}^{2}=M_{+} T_{-} T_{+} M_{+}=M_{+} P M_{+},
$$

where $P=1-\left|e_{0}\right\rangle\left\langle e_{0}\right|$ and $\left|e_{0}\right\rangle\left\langle e_{0}\right|$ is the projector to the zero-th component. This relation implies

$$
\operatorname{Tr} M_{-}^{2 n}=\operatorname{Tr}\left(M_{+}^{2} P\right)^{n}
$$

The right hand side looks similar to (3.18) and therefore suggests that a similar manipulation is possible. All we have to do is to rewrite the right hand side by expanding $P=1-\left|e_{0}\right\rangle\left\langle e_{0}\right|$. 
Again, this is most easily done by studying the determinants. Let us rewrite the right hand side of (4.14). We use the fact $\Xi_{ \pm}(z)=\operatorname{det}\left(1+z M_{ \pm}\right)$, then

$$
\frac{\operatorname{det}\left(1-z^{2} M_{-}^{2}\right)}{\operatorname{det}\left(1-z^{2} M_{+}^{2}\right)}=\frac{\operatorname{det}\left(1-z^{2} M_{+}^{2} P\right)}{\operatorname{det}\left(1-z^{2} M_{+}^{2}\right)}=\operatorname{det}\left(1+\frac{z^{2} M_{+}^{2}}{1-z^{2} M_{+}^{2}}\left|e_{0}\right\rangle\left\langle e_{0}\right|\right) .
$$

From the similar argument to derive (3.23), this determinant becomes simple

$$
\operatorname{det}\left(1+\frac{z^{2} M_{+}^{2}}{1-z^{2} M_{+}^{2}}\left|e_{0}\right\rangle\left\langle e_{0}\right|\right)=1+\left\langle e_{0}\left|\frac{z^{2} M_{+}^{2}}{1-z^{2} M_{+}^{2}}\right| e_{0}\right\rangle=\left\langle e_{0}\left|\frac{1}{1-z^{2} M_{+}^{2}}\right| e_{0}\right\rangle .
$$

Expanding this in $z$, we obtain

$$
\frac{\operatorname{det}\left(1-z^{2} M_{-}^{2}\right)}{\operatorname{det}\left(1-z^{2} M_{+}^{2}\right)}=\sum_{n=0}^{\infty} z^{2 n}\left(M_{+}^{2 n}\right)_{00} .
$$

Now we want to write the right hand side of (4.19) in terms of the coefficients $g_{n}$ of $G(z)$. To do this, we use (2.47) and (3.38). Setting $q=0$ in the second equation of (2.47), we obtain

$$
4 \tilde{\phi}_{+}^{k}(0)=\left(M_{+}^{k-1}\right)_{00} .
$$

On the other hand, if setting $q=0$ in (3.38), we also obtain

$$
4 \tilde{\phi}_{+}^{2 n+1}(0)=\sum_{m=0}^{2 n}(-1)^{m} g_{m} g_{2 n-m} .
$$

where we have used (3.37). Comparing these relations, we find

$$
\left(M_{+}^{2 n}\right)_{00}=\sum_{m=0}^{2 n}(-1)^{m} g_{m} g_{2 n-m} .
$$

Substituting (4.22) into (4.19), one can confirm that (4.14) really holds.

\section{$5 \quad$ Numerical results}

In the previous section, we compute the (grand) partition function analytically. Here we discuss another approach to analyze the partition function of the ABJM theory. The key idea is to use a link between the Fredholm theory for the kernel like (2.4) and TBA system. This novel link was first considered by Zamolodchikov to study two-dimensional polymers in [25]. The kernel appearing there is similar to (2.4). Thus we can use some of the results in [25]. Particularly, we can write down the TBA-type integral equations that give $\operatorname{Tr} \rho^{n}$. Though the TBA equations can not be solved analytically in general, it is easy to solve them at least 
numerically. In this section, we numerically solve the TBA-like equations and compute $\operatorname{Tr} \rho^{n}$. We compare them with the exact results in the previous section. Our numerical results show the very good agreement with the exact ones, and further give a prediction of $\operatorname{Tr} \rho^{n}$ (and also the partition function), of which we have not found the exact values, with a very high degree of accuracy. We also study numerically the eigenvalue spectrum of the density matrix.

\subsection{Partition function from TBA-like equations}

In order to see the relation to the TBA-like equations, it is convenient to rewrite the kernel (2.4) as the form

$$
\rho\left(q_{1}, q_{2}\right)=\frac{e^{-\frac{1}{2} U\left(q_{1}\right)-\frac{1}{2} U\left(q_{2}\right)}}{2 \cosh \left(\frac{q_{1}-q_{2}}{2 k}\right)}
$$

where

$$
U(q)=\log \left[2 \cosh \frac{q}{2}\right]
$$

In [25], Zamolodchikov considered the following integral

$$
R_{n}(x)=e^{-2 u(x)} \int_{-\infty}^{\infty} \cdots \int_{-\infty}^{\infty} d x_{1} \cdots d x_{n} \frac{e^{-2 u\left(x_{1}\right)-\cdots-2 u\left(x_{n}\right)}}{\cosh \left(\frac{x-x_{1}}{2}\right) \cosh \left(\frac{x_{1}-x_{2}}{2}\right) \cdots \cosh \left(\frac{x_{n}-x}{2}\right)}
$$

One can easily check that if $u(x)=U(k x) / 2$ is chosen, the integral of $R_{n}(x)$ is related to $\operatorname{Tr} \rho^{n+1}$ thorough

$$
\int_{-\infty}^{\infty} d x R_{n}(x)=(4 \pi)^{n+1} \operatorname{Tr} \rho^{n+1}
$$

Let us introduce the generating functionals of $R_{n}(x)$,

$$
\begin{aligned}
R_{e}(x \mid z) & =\sum_{m=0}^{\infty}\left(-\frac{z}{4 \pi}\right)^{2 m} R_{2 m}(x), \quad R_{o}(x \mid z)=\sum_{m=0}^{\infty}\left(-\frac{z}{4 \pi}\right)^{2 m+1} R_{2 m+1}(x), \\
R(x \mid z) & =R_{e}(x \mid z)+R_{o}(x \mid z)
\end{aligned}
$$

From (2.6) and (5.4), we obtain $\left(z=e^{\mu}\right)$

$$
\frac{1}{4 \pi} \int_{-\infty}^{\infty} d x R(x \mid z)=\frac{d}{d z} \log \Xi(z)=e^{-\mu} J^{\prime}(\mu)
$$

where $J(\mu)=\log \Xi(z)$ is the grand potential. Thus if we know the function $R(x \mid z)$, we can obtain the grand partition function. 
One of the important results in [25] is that the function $R_{e}(x \mid z)$ is conjectured to be the solution of the following TBA-type integral equations

$$
\begin{aligned}
\log R_{e}(x \mid z) & =\log s(k x)+\int_{-\infty}^{\infty} d x^{\prime} K_{1}\left(x-x^{\prime}\right) \log \left(1+\eta^{2}\left(x^{\prime} \mid z\right)\right) \\
\eta(x \mid z) & =-z \int_{-\infty}^{\infty} d x^{\prime} K_{1}\left(x-x^{\prime}\right) R_{e}\left(x^{\prime} \mid z\right)
\end{aligned}
$$

where $\eta(x \mid z)$ is an auxiliary function, and we have introduced the following functions

$$
s(x)=\frac{1}{2 \cosh \frac{x}{2}}, \quad K_{1}(x)=\frac{1}{2 \pi \cosh x} .
$$

These two equations determine the functions $R_{e}(x \mid z)$ and $\eta(x \mid z)$ uniquely for given $z$. Once $R_{e}(x \mid z)$ and $\eta(x \mid z)$ are determined, the function $R_{o}(x \mid z)$ is given by

$$
R_{o}(x \mid z)=R_{e}(x \mid z) \int_{-\infty}^{\infty} d x^{\prime} K_{2}\left(x-x^{\prime}\right) \arctan \eta\left(x^{\prime} \mid z\right)
$$

where

$$
K_{2}(x)=\frac{1}{\pi \cosh ^{2} x} .
$$

Therefore we can obtain the whole function $R(x \mid z)$ from these integral equations. In our case, the driving term in (5.8) takes the form $-\log (2 \cosh (k x / 2))$, which is not a standard form $-m \cosh x$ in ordinary TBA systems for $2 \mathrm{~d}$ relativistic quantum field theories. Note that this conjecture has been proved in [24].

\subsection{Numerical results from TBA}

Now we want to solve the TBA-like equations (5.8), (5.9) and (5.11) and to compute $\operatorname{Tr} \rho^{n}$. For this purpose, we expand $\eta(x \mid z)$ as

$$
\eta(x \mid z)=\sum_{m=0}^{\infty}\left(-\frac{z}{4 \pi}\right)^{2 m+1} \eta_{2 m+1}(x) .
$$

Substituting (5.5) and (5.13) into (5.8), we obtain the following first few equations,

$$
\begin{aligned}
& \log R_{0}(x)=\log s(k x), \quad \frac{R_{2}(x)}{R_{0}(x)}=K_{1} * \eta_{1}^{2}, \\
& \frac{R_{4}(x)}{R_{0}(x)}-\frac{1}{2}\left(\frac{R_{2}(x)}{R_{0}(x)}\right)^{2}=K_{1} *\left(2 \eta_{1} \eta_{3}-\frac{\eta_{1}^{4}}{2}\right),
\end{aligned}
$$


where the convolution is defined by

$$
f * g=\int_{-\infty}^{\infty} d x^{\prime} f\left(x-x^{\prime}\right) g\left(x^{\prime}\right)
$$

Similarly, from (5.9), we obtain

$$
\eta_{2 m+1}(x)=4 \pi K_{1} * R_{2 m}
$$

for $m=0,1, \cdots$. Starting with $R_{0}(x)=s(k x)$, one can determine $\eta_{1}(x)$ from (5.16), then $R_{2}(x)$ is determined by the second equation in (5.14). The flow of the determined functions is as follows,

$$
R_{0}(x) \rightarrow \eta_{1}(x) \rightarrow R_{2}(x) \rightarrow \eta_{3}(x) \rightarrow R_{4}(x) \rightarrow \cdots
$$

In the similar way, $R_{n}(x)$ for odd $n$ is fixed from (5.11) such as

$$
R_{1}(x)=R_{0}(x) K_{2} * \eta_{1}, \quad R_{3}(x)=R_{0}(x) K_{2} *\left(\eta_{3}-\frac{\eta_{1}^{3}}{3}\right)+R_{2}(x) K_{2} * \eta_{1} .
$$

The integral equations (5.14), (5.16), (5.18) etc. are solved recursively. In principle, we can solve them analytically by evaluating the convolutions of the known functions at each step. Technically, however, it is very messy to do that. Alternatively, here we solve these sequential integral equations numerically at $k=13$ If $R_{n}(x)$ is determined, we can compute $\operatorname{Tr} \rho^{n+1}$ from (5.4).

We have indeed computed the numerical values of $\operatorname{Tr} \rho^{n}$ from such integral equations. In Table 1, we summarize the numerical values for $2 \leq n \leq 16$. The numerical results show the very good agreement with the exact values obtained in the previous section. We would like to emphasize that the TBA-like equations are valid for general $k$ (even for non-integer values). The only difference is the initial condition $R_{0}(x)=s(k x)$. We can predict the high-accurate values of the partition functions for various $N$ and $k$ in the same manner.

In this subsection, we expand all the unknown functions $R_{e}(x \mid z), R_{o}(x \mid z)$ and $\eta(x \mid z)$ in $z$ to compute $\operatorname{Tr} \rho^{n}$. We note that the TBA-like equations (5.8), (5.9) and (5.11) can be also solved for given $z$ by iteration as we will see in the next section.

\subsection{Eigenvalue distribution}

Here we study the eigenvalue distribution of the density matrix numerically. For this purpose, we use the Hankel matrix representation discussed in Section 2.2. Since this matrix is discrete,

\footnotetext{
${ }^{3}$ We have also analytically checked that the TBA-like equations indeed reproduce the correct $\operatorname{Tr} \rho^{n}$ up to $n=5$.
} 
Table 1: Numerical values of $(2 \pi)^{n} \operatorname{Tr} \rho^{n}$ at $k=1$. These numerical values are computed by using the TBA-like equations (5.8), (5.9) and (5.11). Our numerical results nicely agree with the exact values in the previous section.

\begin{tabular}{ccc}
\hline$n$ & $(2 \pi)^{n} \operatorname{Tr} \rho^{n}$ & Errors \\
\hline 2 & 0.896604773477443035477301058329286341729730 & $1.1 \times 10^{-40}$ \\
3 & 0.698731738515872159486421033736470898141922 & $1.7 \times 10^{-40}$ \\
4 & 0.587330256906414478771564191224945948765499 & $2.1 \times 10^{-40}$ \\
5 & 0.505385252214593561502730078447293192743804 & $2.2 \times 10^{-40}$ \\
6 & 0.438346955317146787321292739552212309497233 & $2.3 \times 10^{-40}$ \\
7 & 0.381274926859615816279007202371073982670788 & $2.2 \times 10^{-40}$ \\
8 & 0.331972050713896807160802466534622236608778 & $2.2 \times 10^{-40}$ \\
9 & 0.289152279521382289292363666349330742224258 & $2.0 \times 10^{-40}$ \\
10 & 0.251890109244736461833445267225791497711006 & \\
11 & 0.219440841867035365985490063842951367327288 & $1.7 \times 10^{-40}$ \\
12 & 0.191175332952772245351675601074802777111664 & \\
13 & 0.166551755978085254984697038651649345830601 & $1.4 \times 10^{-40}$ \\
14 & 0.145100086034249146678243847815416397520091 & \\
15 & 0.126411483244355445082928632866179620259652 & \\
16 & 0.110129973053738524406779285719767961758381 & \\
\hline
\end{tabular}

we can evaluate its eigenvalues by the level truncation. Furthermore the Hankel matrix elements $M_{i j}$ only depend on $i+j$. This means that we can reduce the $\mathcal{O}\left(L^{2}\right)$-computation into $\mathcal{O}(L)$-computation where $L$ is the size of the matrix. This is the reason why we use the Hankel matrix $M$ rather than the Chebyshev representation $M^{\rho}$ of the density matrix. Physically, we are interested in the energy spectrum of the Fermi gas system. Since the Hamiltonian is related to the density matrix as $\rho=e^{-H}$, the $n$-th energy eigenvalue is given by

$$
E_{n}=-\log \lambda_{n}
$$

where $\lambda_{n}$ is the eigenvalue of the density matrix.

In Table 2, we summarize the numerical result of the energy eigenvalues of this quantum mechanical system, computed from the Hankel matrix $M$ using the level truncation. In Figure 1, we show the distribution of these energy eigenvalues. We have used the extrapolated values 
Table 2: Numerical energy eigenvalues of the Fermi gas system at $k=1$. The values at $\infty$ are obtained by extrapolating the data with the functions $e^{(0)}+e^{(1)} / L+e^{(2)} / L^{2}+e^{(3)} / L^{3}$ where $L$ is the size of the matrix. $E_{ \pm, n}$ mean the $n$-th energy eigenvalues of the systems described by the density matrices $\rho_{ \pm}$.

\begin{tabular}{cccccccc}
\hline Size & $E_{0}\left(E_{+, 0}\right)$ & $E_{1}\left(E_{-, 0}\right)$ & $E_{2}\left(E_{+, 1}\right)$ & $E_{3}\left(E_{-, 1}\right)$ & $E_{4}\left(E_{+, 2}\right)$ & $E_{5}\left(E_{-, 2}\right)$ & $E_{6}\left(E_{+, 3}\right)$ \\
\hline 100 & 1.975939 & 2.977194 & 3.739240 & 4.432293 & 5.142744 & 5.895999 & 6.709097 \\
200 & 1.975808 & 2.974644 & 3.721872 & 4.372444 & 5.003785 & 5.656839 & 6.350546 \\
400 & 1.975772 & 2.973812 & 3.715146 & 4.344395 & 4.928247 & 5.510457 & 6.116287 \\
1000 & 1.975760 & 2.973523 & 3.712410 & 4.330522 & 4.883362 & 5.408403 & 5.933915 \\
2000 & 1.975759 & 2.973474 & 3.711873 & 4.327247 & 4.870444 & 5.372886 & 5.860046 \\
4000 & 1.975758 & 2.973460 & 3.711711 & 4.326134 & 4.865368 & 5.356542 & 5.820704 \\
10000 & 1.975758 & 2.973456 & 3.711658 & 4.325733 & 4.863290 & 5.348715 & 5.798487 \\
$\infty$ & 1.975758 & 2.973449 & 3.711541 & 4.324808 & 4.859445 & 5.339096 & 5.782347 \\
\hline
\end{tabular}

as in Table 2 to plot this graph. We observe that the square of the energy eigenvalue $E_{n}$ shows the good linear behavior on the excitation level $n$ for small $n$ :

$$
E_{n}^{2}-E_{0}^{2} \approx \frac{\pi^{2}}{2} n
$$

Surprisingly, the coefficient of $n$ in this equation agrees with the one appearing in the relation between the number of states and the energy in the thermodynamic limit [17]: $n(E)=\frac{2}{\pi^{2} k} E^{2}+$ $n_{0}$.

Let us compare these results with the estimations from the exact results in the previous section. We can estimate the ground state and the first excited state energies from $\operatorname{Tr} \rho_{ \pm}^{n}$ because

$$
\operatorname{Tr} \rho_{ \pm}^{n}=\sum_{\ell=0}^{\infty} \lambda_{ \pm, \ell}^{n} \approx \lambda_{ \pm, 0}^{n}
$$

for large $n$. Using (4.8) and (C.7), we find

$$
E_{0}=E_{+, 0} \approx-\log \left(\frac{\operatorname{Tr} \rho_{+}^{8}}{\operatorname{Tr} \rho_{+}^{7}}\right)=1.9757623 \cdots,
$$

which matches the estimation from the Hankel matrix in Table 2. The order of the subleading 


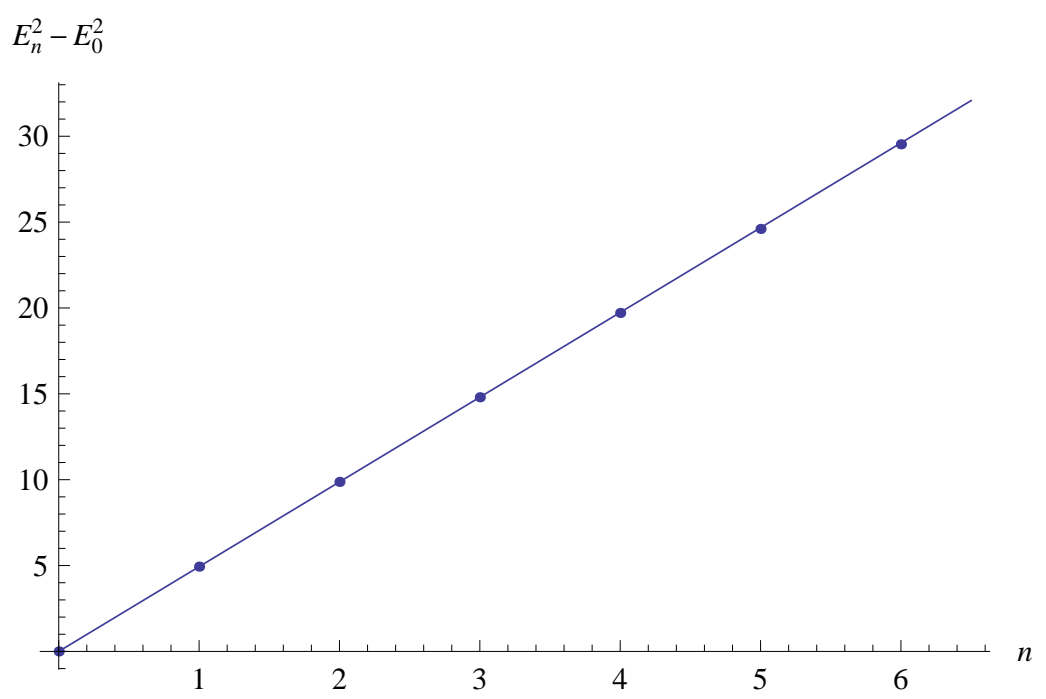

Figure 1: The (square of) energy spectrum of the Fermi gas system against the excitation level $n$. The dots correspond to the extrapolated numerical eigenvalues in Table 2 while the solid line is the plot of $E^{2}-E_{0}^{2}=\pi^{2} n / 2$.

corrections is $\mathcal{O}\left(\lambda_{2}^{7} / \lambda_{0}^{7}\right) \sim \mathcal{O}\left(10^{-6}\right)$. Similarly,

$$
E_{1}=E_{-, 0} \approx-\log \left(\frac{\operatorname{Tr} \rho_{-}^{8}}{\operatorname{Tr} \rho_{-}^{7}}\right)=2.9735127 \cdots,
$$

where we have used the exact results (4.9) and (C.8). The result is again consistent with the value in Table 2, The subleading corrections come from $\mathcal{O}\left(\lambda_{3}^{7} / \lambda_{1}^{7}\right) \sim \mathcal{O}\left(10^{-5}\right)$.

\section{Comparison with perturbative results}

In this section, we compare our results with the perturbative ones in [17].

We first consider the free energy of the ABJM theory. In [15, 17], it was shown that the ABJM partition function is written in terms of the Airy function if one neglects the instanton corrections. Since we have obtained the exact partition function $Z(N)$ up to $N=9$ in Section 4, we can compare them with the perturbative results. This comparison allows us to know the behavior of the non-perturbative effects. Our results suggest that the leading non-perturbative correction scales as $e^{-2 \pi \sqrt{2 N}}$.

We next consider the expectation value of $N$ (or the derivative of the grand potential). Using the TBA-like equations in the previous section, we can numerically compute $\langle N\rangle$ as a 
function of the chemical potential $\mu$. Our numerical results show the good agreement with the perturbative solution in [17] in the large $\mu$ limit.

\subsection{Free energy}

Let us consider the free energy of the ABJM theory. As was shown in [15, 17], the partition function is written in terms of the Airy function if one neglects the instanton corrections,

$$
\begin{aligned}
Z_{k}(N) & =Z_{k}^{(\text {Airy })}(N)+Z_{k}^{(\mathrm{np})}(N) \\
Z_{k}^{(\text {Airy })}(N) & =\left(\frac{2}{\pi^{2} k}\right)^{-1 / 3} e^{A(k)} \mathrm{Ai}\left[\left(\frac{2}{\pi^{2} k}\right)^{-1 / 3}\left(N-\frac{1}{3 k}-\frac{k}{24}\right)\right]
\end{aligned}
$$

where $Z_{k}^{(\mathrm{np})}(N)$ is the non-perturbative contribution. The function $A(k)$ has the following small $k$ expansion,

$$
A(k)=\frac{2 \zeta(3)}{\pi^{2} k}-\frac{k}{12}-\frac{\pi^{2} k^{3}}{4320}+\mathcal{O}\left(k^{5}\right) .
$$

In [31], it was conjectured that this function has the following integral expression, which should be valid for arbitrary $k$,

$$
\begin{aligned}
A(k)= & \frac{1}{2} \log 2-\frac{\zeta(3)}{8 \pi^{2}} k^{2}-\frac{1}{6} \log k+\frac{1}{6} \log \left(\frac{\pi}{2}\right)+2 \zeta^{\prime}(-1) \\
& -\frac{1}{3} \int_{0}^{\infty} d x \frac{1}{e^{k x}-1}\left(\frac{3}{x^{3}}-\frac{1}{x}-\frac{3}{x \sinh ^{2} x}\right) .
\end{aligned}
$$

Figure 2 (a) shows the behavior of the free energy $F(N)=-\log Z(N)$ at $k=1$. The result (6.2) shows the very close behavior of the exact results even though the non-perturbative corrections are dropped. It is surprising that these corrections at $k=1$ is very small even for small $N$.

Let us consider the non-perturbative corrections to the free energy

$$
F_{1}^{(\mathrm{np})}(N) \equiv F_{1}^{(\text {exact })}(N)-F_{1}^{(\text {Airy })}(N),
$$

where $\left.F_{k}^{(\text {Airy) }}(N)=-\log Z_{k}^{(\text {Airy) }}(N) 4\right]$ We find $F_{1}^{(\mathrm{np})}(N)$ is negative for all $N \leq 9$. In [14, 17] it was noticed that there are two kinds of non-perturbative instanton effects contributing to the free energy. One of them is the worldsheet instanton $\exp (-2 \pi \sqrt{2 N / k})$, while the other is the membrane instanton $\exp (-\pi \sqrt{2 k N})$ which comes from D2-branes wrapping on $\mathbb{R} P^{3}$ in $\mathbb{C} P^{3}$. Since in the case $k=1$ there is no $\mathbb{R} P^{3}$ to wrap, we expect that the membrane instanton effect vanishes and the non-perturbative corrections start from $\exp (-2 \pi \sqrt{2 N})$.

\footnotetext{
${ }^{4}$ In the definition here, $F_{k}^{(\mathrm{np})}(N) \neq-\log Z_{k}^{(\mathrm{np})}(N)$ where $Z_{k}^{(\mathrm{np})}(N)=Z_{k}^{(\text {exact })}(N)-Z_{k}^{(\text {Airy })}(N)$.
} 


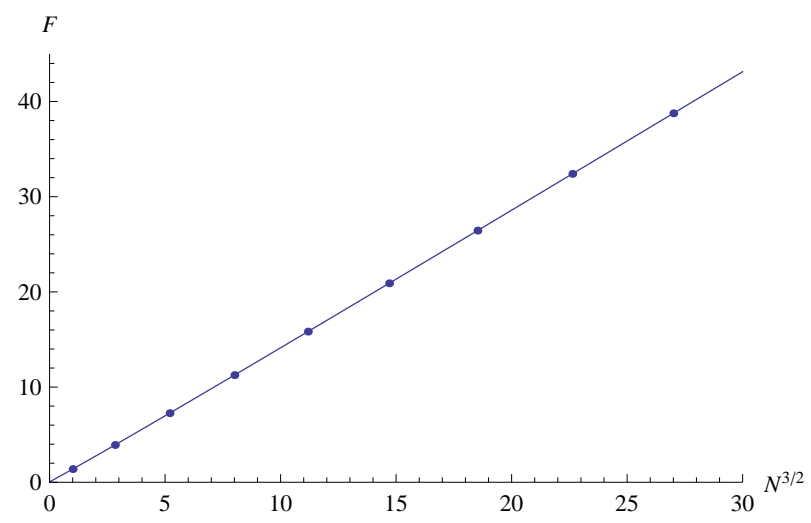

(a)

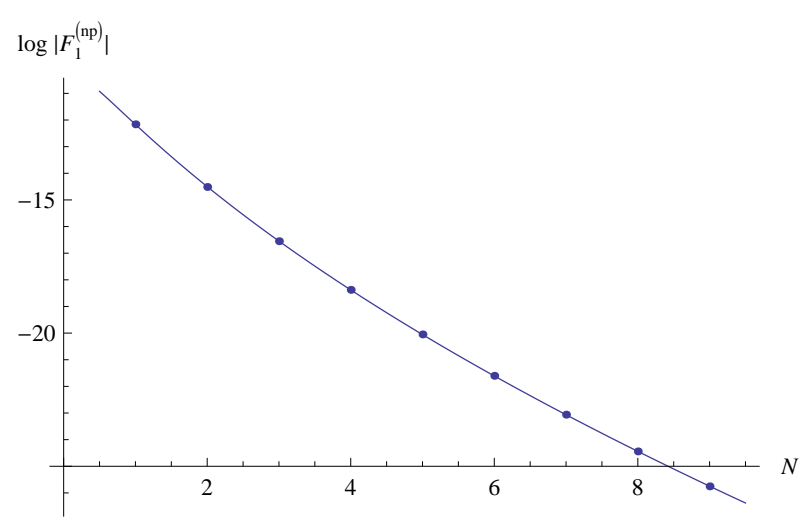

(b)

Figure 2: The behavior of the free energy at $k=1$. (a) The dots show our exact values while the solid line shows the free energy computed from (6.2). (b) The (log of) non-perturbative correction $F_{1}^{(\mathrm{np})}(N)$. The dots are the exact data, and the solid line is the fitted function (6.6) with (6.7).

In Figure 2 (b), we tried to fit our exact data of $\log \left|F_{1}^{(\mathrm{np})}(N)\right|$ by the function

$$
\log \left|F_{1}^{(\mathrm{np})}(N)\right| \sim a+b \log N-c \sqrt{N}
$$

and found the best values of the fitting

$$
a=-3.17, \quad b=2.01, \quad c=9.00 \text {. }
$$

Note especially that the value of $c$ is very close to $2 \pi \sqrt{2}=8.88577$. This result strongly suggests that the membrane instanton $e^{-\pi \sqrt{2 k N}}$ is absent when $k=1$, and the leading nonperturbative correction behaves as $F_{1}^{\mathrm{np}}(N) \sim e^{-2 \pi \sqrt{2 N}}$ at large $N$.

Finally, let us briefly comment on the pre-factor of the instanton correction $e^{-2 \pi \sqrt{2 N}}$. From the general argument in [17], one expects that $F_{1}^{(\mathrm{np})}(N)$ scales as

$$
F_{1}^{(\mathrm{np})}(N) \sim(A N+B \sqrt{N}+C) e^{-2 \pi \sqrt{2 N}}
$$

with some coefficients $A, B$ and $C$. Our analysis (6.7) seems to indicate that the leading term of $F_{1}^{(\mathrm{np})}(N)$ behaves as $N^{2} e^{-2 \pi \sqrt{2 N}}$ rather than $N e^{-2 \pi \sqrt{2 N}}$. However, our result up to $N=9$ is insufficient to tell. One possibility is that $F_{1}^{(\mathrm{np})}(N)$ may actually scale as (6.8) for much larger $N$ than $N=9$. It would be interesting to study the behavior of the non-perturbative correction $F_{1}^{(\mathrm{np})}(N)$ in more detail. 


\subsection{Expectation value of $N$}

In the previous section, we solved the TBA-like equations by expanding all the unknown functions in $z$ in order to compute $\operatorname{Tr} \rho^{n}$. It is possible to solve them for given $z$ by iteration. Let us compare the results from such integral equations with the perturbative ones as functions of $z=e^{\mu}$. For this purpose, it is convenient to focus on the derivative of the grand potential $J(\mu)$ because this quantity naturally appears in the TBA approach as in (5.7). From the standard argument of statistical mechanics, the derivative $J^{\prime}(\mu)$ corresponds to the expectation value of $N$ in the grand canonical ensemble

$$
\langle N\rangle=J^{\prime}(\mu)=\frac{e^{\mu}}{4 \pi} \int_{-\infty}^{\infty} d x R\left(x \mid e^{\mu}\right),
$$

where (5.7) has been used in the last equality. Using (2.4), the grand potential is also expressed in terms of $\operatorname{Tr} \rho^{n}$. The expectation value $\langle N\rangle$ is thus written as the expansion around $\mu=-\infty$

$$
\langle N\rangle=-\sum_{n=1}^{\infty}\left(-e^{\mu}\right)^{n} \operatorname{Tr} \rho^{n} .
$$

Let us consider the perturbative contribution of $J(\mu)$. As shown in [17], the grand potential of the ABJM theory is given by

$$
J^{(\text {pert })}(\mu)=\frac{2 \mu^{3}}{3 k \pi^{2}}+\mu\left(\frac{1}{3 k}+\frac{k}{24}\right)+A(k),
$$

if non-perturbative corrections are neglected. Thus the derivative of $J(\mu)$ is given by

$$
\langle N\rangle^{(\text {pert })}=\frac{2 \mu^{2}}{k \pi^{2}}+\frac{1}{3 k}+\frac{k}{24} .
$$

Note that, in (6.12), the contribution from $A(k)$ is dropped.

We numerically solve the TBA-like equations (5.8), (5.9) and (5.11) at $k=1$ for various values of $z=e^{\mu}$, and compute the expectation value of $N$ by using (6.9) as a function of $\mu$. The results are shown in Figure 3. In the figure, the dots represent our numerical results from the TBA-like equations. The red solid line is the plot of the truncated expansion (6.10) up to $n=9$ where we have used the exact values of $\operatorname{Tr} \rho^{n}$. The green dashed line represents the perturbative result (6.12). We find that our numerical results are close to the perturbative result (6.12) in the large $\mu$ limit while in the limit $\mu \rightarrow-\infty$ the expansion (6.10) agrees with the numerical results as expected. The TBA interpolates both results smoothly. This is not surprising because the TBA results contain all the non-perturbative corrections, which are neglected in (6.12). 


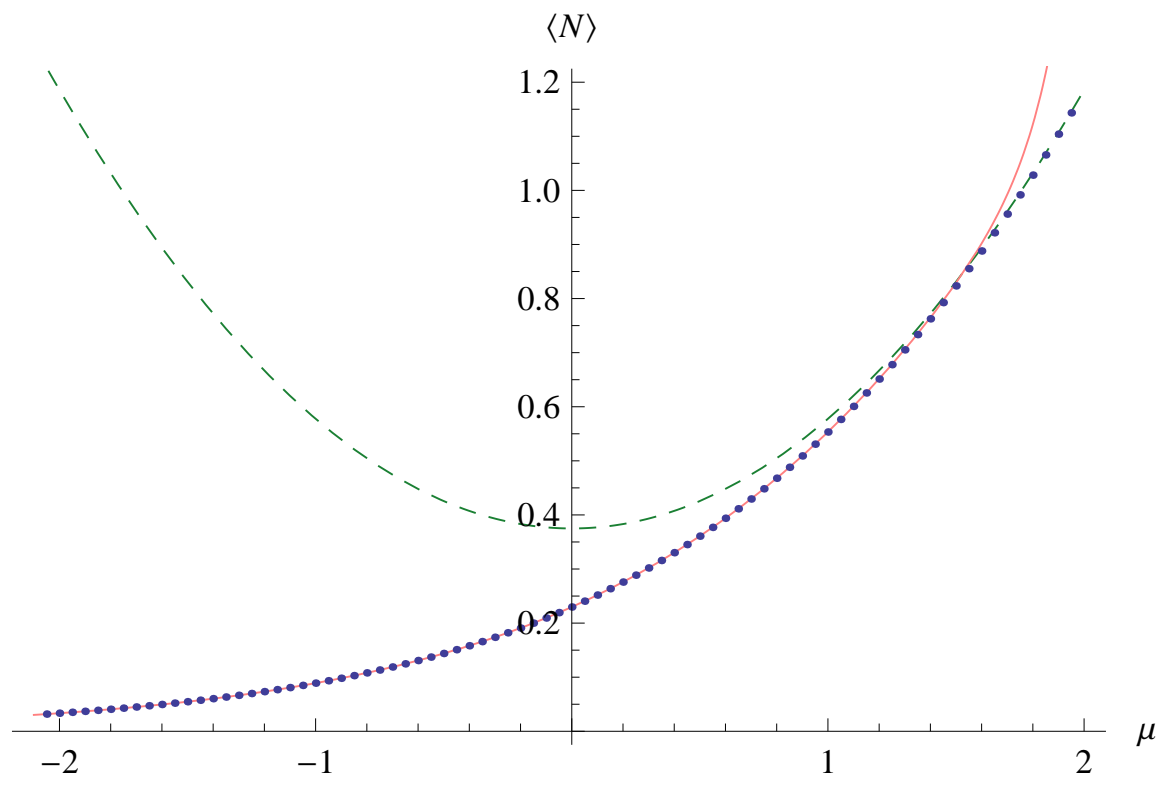

Figure 3: The behavior of the expectation value of $N$ as a function of $\mu$. The dots show the results obtained from TBA-like equations. The red solid line represents the finite-truncated expansion (6.10) up to $n=9$ with the exact $\operatorname{Tr} \rho^{n}$ while the green dashed line represents the perturbative result (6.12).

Finally, let us give a comment on an underlying technical issue in this TBA system. As was mentioned in [25], the iterative solution of the TBA equations describing the Fredholm determinant converges only when $z$ is in the regime $|z|<1 / \lambda_{0}$ where $\lambda_{0}$ is the largest eigenvalue of the kernel $\rho$. If $|z|$ crosses over this value, the iteration method does not work any more. In our interested case $k=1$, the bound of the convergence exists at $|z|=1 / \lambda_{0} \approx 7.21(\mu \approx 1.97)$.

\section{Discussion}

In this paper, we have studied the Fermi gas quantum mechanics associated to the ABJM matrix model. We have computed $\operatorname{Tr} \rho^{n}$, which is the ingredient of the grand partition function, and obtained the partition function $Z(N)$ up to $N=9$ as a result. In reducing the number of integrations by commuting coordinates and momenta, we have found an exact relation concerning the grand partition function, which is interesting by itself and very helpful for determining the partition function. We have also performed various numerical analysis. All of these results are consistent with our exact results and also with the perturbative analysis 
in [17].

Interestingly, all the partition functions we obtained are written in terms of polynomials of $\pi^{-1}$ with rational coefficients. Similarly, the functions $\phi_{ \pm}^{k}(q)$ which lead to the determination of $\operatorname{Tr} \rho^{n}$ are also expressed in terms of polynomials of $\pi^{-1}, q, s_{q}, c_{q}, s_{q / 2}$ and $c_{q / 2}$ with rational coefficients. Since we have computed the partition function exactly and integer numbers (or rational numbers) usually counts some information of the moduli space, we would like to know what kind of the information we are counting right now.

We would like to understand better the origin of the anomaly in commuting the coordinate $q$ and the momentum $p$. From the analogy of string field theory, typically these kinds of anomaly appear because of the singularity of the moduli space we are considering and give some information to the physical problem. For example, the associativity anomaly [32] in cubic string field theory [33] comes from the singularity at the boundary of the moduli space and relates to the translational invariance in string field theory. Also, the twist anomaly [34] in vacuum string field theory [35] originates from the divergence at the midpoint interaction point and contains the information of the tachyon mass and the brane tension.

We have shown that finding the energy spectrum of our Fermi gas system boils down to the diagonalization of the Hankel matrix $M$ defined by (2.39). As in the case of the diagonalization of Neumann matrices in open string field theory [36], the first step would be to find a (hidden) symmetry $K$ commuting with $M:[M, K]=0$. It would be very interesting to find the exact spectrum of $M$ and write down the exact grand partition function.

We showed in (2.13) that the grand partition function of the ABJM theory is factorized into two parts. A similar structure has appeared in the study of holographic dual of BPS black holes [37, and it was argued that going to the fixed charge sector leads to the entanglement of two sectors. Such entangled state has an interpretation of branched tree of baby universes and the Catalan number appears as the number of branchings [37. It would be interesting to see if the factorized grand partition function of the ABJM theory has some interpretation on the bulk gravity side.

We also would like to remark on an analogy with the so-called ODE/IM correspondence [38, 39]. In [40, 41, 42], it was found that spectral determinants for certain Schrödinger equations satisfy functional relations. In [38, 39], these spectral determinants were identified with Baxter's $Q$-functions of the corresponding integrable system, and the functional relations of the spectral determinants just correspond to the quantum Wronskian relations [43]. Our analysis implies that there is a non-trivial relation between the two determinants $\Xi_{ \pm}(z)$ because the total determinant $\Xi(z)$ is fixed by the information of either of the two sectors $\rho_{ \pm}$. It would be interesting to explore whether $\Xi_{ \pm}(z)$ satisfy a functional relation like the quantum 
Wronskian relation or not.

Recently, the expectation values of some BPS Wilson loops of the ABJM theory were analyzed using the Fermi gas formalism [19]. It was found that the semiclassical expansion of such Wilson loop expectation values is divergent for $k=1,2$. We hope that our formalism will give some improvements for the computation of Wilson loops.

\section{Acknowledgements}

We are grateful to Hiroyuki Hata, Masazumi Honda, Kazuo Hosomichi, Hiroaki Kanno, Tomoki Nosaka, Satoru Odake, Ryu Sasaki, Naoki Sasakura for useful discussions. Y.H. would like to thank Junji Suzuki for telling him the Mathematica code to solve TBA equations by using FFT algorithm. Y.H. is grateful to the hospitality of the IPhT, CEA/Saclay and S.M. is grateful to the hospitality of YITP at Kyoto University. The work of Y.H. is supported in part by the JSPS Research Fellowship for Young Scientists, while the work of K.O. is supported in part by JSPS Grant-in-Aid for Young Scientists (B) \#23740178.

\section{A Recurrence relations for $\phi_{ \pm}^{k}(q)$}

Let us derive the recurrence relations (2.25). Using (2.22), we find

$$
\begin{aligned}
\phi_{ \pm}^{k}(q) & =\frac{1}{E_{ \pm}(q)} \int \frac{d q^{\prime}}{2 \pi} \frac{d q^{\prime \prime}}{2 \pi} \rho_{ \pm}\left(q, q^{\prime}\right) \rho_{ \pm}^{k-1}\left(q^{\prime}, q^{\prime \prime}\right) E_{ \pm}\left(q^{\prime \prime}\right) \\
& =\frac{1}{E_{ \pm}(q)} \int \frac{d q^{\prime}}{2 \pi} \rho_{ \pm}\left(q, q^{\prime}\right) E_{ \pm}\left(q^{\prime}\right) \phi_{ \pm}^{k-1}\left(q^{\prime}\right) .
\end{aligned}
$$

Using the identity

$$
\frac{1}{\cosh q+\cosh q^{\prime}}=\frac{1}{2 \cosh \frac{q}{2} \cosh \frac{q^{\prime}}{2}}\left(c_{q-q^{\prime}}+c_{q+q^{\prime}}\right),
$$

we find that the kernel is written as

$$
\begin{aligned}
& E_{+}(q)^{-1} \rho_{+}\left(q, q^{\prime}\right) E_{+}\left(q^{\prime}\right)=\frac{\cosh \frac{q^{\prime}}{2}}{2\left(\cosh q+\cosh q^{\prime}\right)}=c_{q} \frac{c_{q-q^{\prime}}+c_{q-q^{\prime}}}{2} \\
& E_{-}(q)^{-1} \rho_{-}\left(q, q^{\prime}\right) E_{-}\left(q^{\prime}\right)=\frac{\sinh ^{2} \frac{q^{\prime}}{2}}{2 \cosh \frac{q^{\prime}}{2}\left(\cosh q+\cosh q^{\prime}\right)}=c_{q} \frac{c_{q-q^{\prime}}+c_{q+q^{\prime}}}{2} t_{q^{\prime}}^{2} .
\end{aligned}
$$

Since $\phi_{ \pm}^{k}(q)$ 's are even functions of $q$, we get

$$
\begin{aligned}
& \phi_{+}^{k}(q)=\int \frac{d q^{\prime}}{2 \pi} c_{q} \frac{c_{q-q^{\prime}}+c_{q+q^{\prime}}}{2} \phi_{+}^{k-1}\left(q^{\prime}\right)=c_{q} \int \frac{d q^{\prime}}{2 \pi} c_{q-q^{\prime}} \phi_{+}^{k-1}\left(q^{\prime}\right), \\
& \phi_{-}^{k}(q)=\int \frac{d q^{\prime}}{2 \pi} c_{q} \frac{c_{q-q^{\prime}}+c_{q+q^{\prime}}}{2} t_{q^{\prime}}^{2} \phi_{-}^{k-1}\left(q^{\prime}\right)=c_{q} \int \frac{d q^{\prime}}{2 \pi} c_{q-q^{\prime}} t_{q^{\prime}}^{2} \phi_{-}^{k-1}\left(q^{\prime}\right),
\end{aligned}
$$

which are the recurrence relations (2.25). 


\section{B Hankel representations of $\phi_{+}^{k}(q)$ and $\tilde{\phi}_{+}^{k}(q)$}

Let us derive (2.47). We expand $\phi_{+}^{k}(q)$ and $\tilde{\phi}_{+}^{k}(q)$ in $t_{q}=\tanh (q / 2)$

$$
\phi_{+}^{k}(q)=4 c_{q}^{2} \sum_{n=0}^{\infty} t_{q}^{2 n} \alpha_{n}^{(k)}, \quad \tilde{\phi}_{+}^{k}(q)=\frac{c_{q}}{2} \sum_{n=0}^{\infty} t_{q}^{2 n} \beta_{n}^{(k)},
$$

We would like to show

$$
\alpha_{n}^{(k)}=\sum_{m=0}^{\infty}\left(M_{+}^{k}\right)_{n m}, \quad \beta_{n}^{(k)}=\left(M_{+}^{k-1}\right)_{n 0} .
$$

Since $\phi_{+}^{0}(q)=1$ and $\tilde{\phi}_{+}^{1}(q)=c_{q} / 2$, one immediately finds

$$
\alpha_{n}^{(0)}=1, \quad \beta_{n}^{(1)}=\delta_{n 0} .
$$

Let us substitute (B.1) into the recurrence relation (3.33). Using the expansion

$$
c_{q-q^{\prime}}=2 c_{q} c_{q^{\prime}} \sum_{n=0}^{\infty} t_{q}^{n} t_{q^{\prime}}^{n}
$$

we obtain

$$
\phi_{+}^{k}(q)=c_{q} \int \frac{d q^{\prime}}{2 \pi}\left(2 c_{q} c_{q^{\prime}} \sum_{n=0}^{\infty} t_{q}^{n} t_{q^{\prime}}^{n}\right) \cdot 4 c_{q^{\prime}}^{2} \sum_{\ell=0}^{\infty} t_{q^{\prime}}^{2 \ell} \alpha_{\ell}^{(k-1)}
$$

The integral over $q^{\prime}$ causes the Hankel matrix because of (2.29). Thus we find

$$
\phi_{+}^{k}(q)=4 c_{q}^{2} \sum_{\ell, n=0}^{\infty} t_{q}^{2 n}\left(M_{+}\right)_{n \ell} \alpha_{\ell}^{(k-1)}
$$

Comparing this equation with (B.1), we obtain

$$
\alpha_{n}^{(k)}=\sum_{\ell=0}^{\infty}\left(M_{+}\right)_{n \ell} \alpha_{\ell}^{(k-1)} .
$$

From this relation and the initial condition ( $(\overline{\mathrm{B} .3})$, one can show that $\alpha_{n}^{(k)}$ is given by (B.2). The derivation of $\beta_{n}^{(k)}$ is straightforward. 


\section{Explicit forms of $\rho_{ \pm}^{n}\left(q_{1}, q_{2}\right)$}

Let us summarize the explicit forms of $\rho_{ \pm}^{n}\left(q_{1}, q_{2}\right)$ up to $n=4$ :

$$
\begin{aligned}
\rho_{+}^{2}\left(q_{1}, q_{2}\right)= & \frac{1}{16} \frac{1}{\sqrt{\cosh \left(\frac{q_{1}}{2}\right) \cosh \left(\frac{q_{2}}{2}\right)}} \frac{1}{\cosh \left(\frac{q_{1}}{2}\right)+\cosh \left(\frac{q_{2}}{2}\right)}, \\
\rho_{+}^{3}\left(q_{1}, q_{2}\right)= & \frac{1}{16 \pi} \frac{\sqrt{\cosh \left(\frac{q_{1}}{2}\right) \cosh \left(\frac{q_{2}}{2}\right)}}{\cosh q_{1}+\cosh q_{2}}\left(\frac{q_{1}}{\sinh q_{1}}+\frac{q_{2}}{\sinh q_{2}}-\frac{\pi}{2 \cosh \left(\frac{q_{1}}{2}\right) \cosh \left(\frac{q_{2}}{2}\right)}\right), \\
\rho_{+}^{4}\left(q_{1}, q_{2}\right)= & \frac{1}{128 \pi} \frac{1}{\sqrt{\cosh \left(\frac{q_{1}}{2}\right) \cosh \left(\frac{q_{2}}{2}\right)}} \frac{1}{\cosh \left(\frac{q_{1}}{2}\right)-\cosh \left(\frac{q_{2}}{2}\right)}\left(\frac{q_{1}}{\sinh q_{1}}-\frac{q_{2}}{\sinh q_{2}}\right) \\
& +\frac{1}{256} \frac{1}{\cosh ^{3 / 2}\left(\frac{q_{1}}{2}\right) \cosh ^{3 / 2}\left(\frac{q_{2}}{2}\right)},
\end{aligned}
$$

and

$$
\begin{aligned}
& \rho_{-}^{2}\left(q_{1}, q_{2}\right)=\frac{1}{16} \frac{\tanh \left(\frac{q_{1}}{4}\right) \tanh \left(\frac{q_{2}}{4}\right)}{\sqrt{\cosh \left(\frac{q_{1}}{2}\right) \cosh \left(\frac{q_{2}}{2}\right)\left(\cosh \left(\frac{q_{1}}{2}\right)+\cosh \left(\frac{q_{2}}{2}\right)\right)}}, \\
& \rho_{-}^{3}\left(q_{1}, q_{2}\right)=\frac{1}{16 \pi} \frac{\sinh \left(\frac{q_{1}}{2}\right) \sinh \left(\frac{q_{2}}{2}\right)}{\sqrt{\cosh \left(\frac{q_{1}}{2}\right) \cosh \left(\frac{q_{2}}{2}\right)}\left(\cosh q_{1}+\cosh q_{2}\right)} \\
& \times\left(\frac{q_{1}}{\sinh q_{1}}+\frac{q_{2}}{\sinh q_{2}}-\frac{\pi}{2 \cosh \left(\frac{q_{1}}{2}\right) \cosh \left(\frac{q_{2}}{2}\right)}\right) \\
& -\frac{1}{64} \frac{\tanh \left(\frac{q_{1}}{4}\right) \tanh \left(\frac{q_{2}}{4}\right)}{\cosh ^{3 / 2}\left(\frac{q_{1}}{2}\right) \cosh ^{3 / 2}\left(\frac{q_{2}}{2}\right)} \\
& \rho_{-}^{4}\left(q_{1}, q_{2}\right)=\frac{1}{256 \pi} \frac{\tanh \left(\frac{q_{1}}{4}\right) \tanh \left(\frac{q_{2}}{4}\right)}{\sqrt{\cosh \left(\frac{q_{1}}{2}\right) \cosh \left(\frac{q_{2}}{2}\right)}}\left(\frac{1}{\sinh ^{2}\left(\frac{q_{1}}{4}\right) \sinh ^{2}\left(\frac{q_{2}}{4}\right)}-\frac{\pi}{\cosh \left(\frac{q_{1}}{2}\right) \cosh \left(\frac{q_{2}}{2}\right)}\right) \\
& +\frac{1}{512 \pi} \frac{\tanh \left(\frac{q_{1}}{4}\right) \tanh \left(\frac{q_{2}}{4}\right)}{\sqrt{\cosh \left(\frac{q_{1}}{2}\right) \cosh \left(\frac{q_{2}}{2}\right)\left(\cosh \left(\frac{q_{1}}{2}\right)-\cosh \left(\frac{q_{2}}{2}\right)\right)}} \\
& \times\left(\frac{q_{1} \operatorname{coth}\left(\frac{q_{1}}{4}\right)}{\cosh \left(\frac{q_{1}}{2}\right) \sinh ^{2}\left(\frac{q_{1}}{4}\right)}-\frac{q_{2} \operatorname{coth}\left(\frac{q_{2}}{4}\right)}{\cosh \left(\frac{q_{2}}{2}\right) \sinh ^{2}\left(\frac{q_{2}}{4}\right)}\right) .
\end{aligned}
$$

These matrix elements are directly derived from (4.3), (4.4) by using (2.23) and (2.24) 5 Using these matrix elements, we can also compute $\operatorname{Tr} \rho_{ \pm}^{2 n+1}$ up to $n=3$ in addition to $\operatorname{Tr} \rho_{ \pm}^{2 n}$ as given in (4.8) and (4.9). The results are given by

$$
\begin{aligned}
& \operatorname{Tr} \rho_{+}=\frac{\sqrt{2}}{8}, \quad \operatorname{Tr} \rho_{+}^{3}=\frac{3-2 \sqrt{2}}{64}, \quad \operatorname{Tr} \rho_{+}^{5}=\frac{(5-8 \sqrt{2}) \pi+20}{2^{10} \pi}, \\
& \operatorname{Tr} \rho_{+}^{7}=\frac{(16 \sqrt{2}-21) \pi^{2}-14 \pi+28}{2^{13} \pi^{2}},
\end{aligned}
$$

5 Similarly we also obtain $\rho_{ \pm}^{n}\left(q_{1}, q_{2}\right)$ for $n=5,6,7$. Since it can be obtained without difficulty, we shall omit their expressions here due to the complexity. 
and

$$
\begin{aligned}
& \operatorname{Tr} \rho_{-}=\frac{2-\sqrt{2}}{8}, \quad \operatorname{Tr} \rho_{-}^{3}=\frac{(1+2 \sqrt{2}) \pi-12}{64 \pi}, \quad \operatorname{Tr} \rho_{-}^{5}=\frac{(8 \sqrt{2}-9) \pi^{2}-20 \pi+40}{2^{10} \pi^{2}}, \\
& \operatorname{Tr} \rho_{-}^{7}=\frac{-9(16 \sqrt{2}+3) \pi^{3}+518 \pi^{2}+756 \pi-336}{2^{13} \cdot 3^{2} \pi^{3}} .
\end{aligned}
$$

Interestingly, in the above results the terms with $\sqrt{2}$ appear. Such terms are canceled out in $\operatorname{Tr} \rho^{2 n+1}$, and $\operatorname{Tr} \rho^{2 n+1}$ do not contain them.

\section{References}

[1] I. R. Klebanov and A. A. Tseytlin, "Entropy of near extremal black p-branes," Nucl. Phys. B 475, 164 (1996) hep-th/9604089.

[2] O. Aharony, O. Bergman, D. L. Jafferis, J. Maldacena, "N=6 superconformal ChernSimons-matter theories, M2-branes and their gravity duals," JHEP 0810, 091 (2008). arXiv:0806.1218 [hep-th]].

[3] O. Aharony, O. Bergman and D. L. Jafferis, "Fractional M2-branes," JHEP 0811, 043 (2008) arXiv:0807.4924 [hep-th]].

[4] M. K. Benna, I. R. Klebanov and T. Klose, "Charges of Monopole Operators in ChernSimons Yang-Mills Theory," JHEP 1001, 110 (2010) [arXiv:0906.3008 [hep-th]].

[5] A. Gustavsson and S. -J. Rey, "Enhanced N=8 Supersymmetry of ABJM Theory on R**8

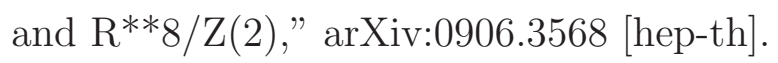

[6] O-K. Kwon, P. Oh and J. Sohn, "Notes on Supersymmetry Enhancement of ABJM Theory," JHEP 0908, 093 (2009) arXiv:0906.4333 [hep-th]].

[7] D. Bashkirov and A. Kapustin, "Supersymmetry enhancement by monopole operators," JHEP 1105, 015 (2011) arXiv:1007.4861 [hep-th]].

[8] A. Kapustin, B. Willett, I. Yaakov, "Exact Results for Wilson Loops in Superconformal Chern-Simons Theories with Matter," JHEP 1003, 089 (2010). arXiv:0909.4559 [hepth]].

[9] V. Pestun, "Localization of gauge theory on a four-sphere and supersymmetric Wilson loops," arXiv:0712.2824 [hep-th]]. 
[10] N. Halmagyi and V. Yasnov, "The Spectral curve of the lens space matrix model," JHEP 0911, 104 (2009) hep-th/0311117.

[11] N. Drukker, M. Marino, P. Putrov, "From weak to strong coupling in ABJM theory," Commun. Math. Phys. 306, 511-563 (2011). arXiv:1007.3837 [hep-th]].

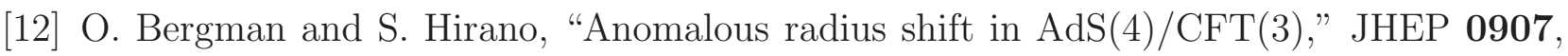
016 (2009) arXiv:0902.1743 [hep-th]].

[13] M. Bershadsky, S. Cecotti, H. Ooguri and C. Vafa, "Kodaira-Spencer theory of gravity and exact results for quantum string amplitudes," Commun. Math. Phys. 165, 311 (1994) hep-th/9309140.

[14] N. Drukker, M. Marino, P. Putrov, "Nonperturbative aspects of ABJM theory," arXiv:1103.4844 [hep-th]].

[15] H. Fuji, S. Hirano, S. Moriyama, "Summing Up All Genus Free Energy of ABJM Matrix Model," JHEP 1108, 001 (2011). arXiv:1106.4631 [hep-th]].

[16] H. Ooguri, C. Vafa and E. P. Verlinde, "Hartle-Hawking wave-function for flux compactifications," Lett. Math. Phys. 74, 311 (2005) hep-th/0502211.

[17] M. Marino and P. Putrov, "ABJM theory as a Fermi gas," J. Stat. Mech. 1203, P03001 (2012) arXiv:1110.4066 [hep-th]].

[18] M. Marino and P. Putrov, "Interacting fermions and N=2 Chern-Simons-matter theories," arXiv:1206.6346 [hep-th].

[19] A. Klemm, M. Marino, M. Schiereck and M. Soroush, "ABJM Wilson loops in the Fermi gas approach," arXiv:1207.0611 [hep-th].

[20] H. Lin, O. Lunin and J. M. Maldacena, "Bubbling AdS space and 1/2 BPS geometries," JHEP 0410, 025 (2004) hep-th/0409174.

[21] S. N. M. Ruijsenaars and H. Schneider, "A New Class Of Integrable Systems And Its Relation To Solitons," Annals Phys. 170, 370 (1986).

[22] S. Odake and R. Sasaki, "Discrete Quantum Mechanics," J. Phys. A A 44, 353001 (2011) arXiv:1104.0473 [math-ph]].

[23] K. Okuyama, "A Note on the Partition Function of ABJM theory on $S^{3}$," Prog. Theor. Phys. 127, 229 (2012) [arXiv:1110.3555 [hep-th]]. 
[24] C. A. Tracy and H. Widom, "Proofs of Two Conjectures Related to the Thermodynamic Bethe Ansatz" Commun.Math.Phys. 179 (1996) 667-680 solv-int/9509003.

[25] A. B. Zamolodchikov, "Painleve III and 2-d polymers," Nucl. Phys. B 432, 427 (1994) hep-th/9409108.

[26] C. P. Herzog, I. R. Klebanov, S. S. Pufu and T. Tesileanu, "Multi-Matrix Models and Tri-Sasaki Einstein Spaces," Phys. Rev. D 83, 046001 (2011) [arXiv:1011.5487 [hep-th]].

[27] A. Kapustin, B. Willett, I. Yaakov, "Nonperturbative Tests of Three-Dimensional Dualities," JHEP 1010, 013 (2010). arXiv:1003.5694 [hep-th]].

[28] I. K. Kostov, "Solvable statistical models on a random lattice," Nucl. Phys. Proc. Suppl. 45A, 13 (1996) hep-th/9509124].

[29] V. A. Kazakov, I. K. Kostov, N. A. Nekrasov, "D particles, matrix integrals and KP hierarchy," Nucl. Phys. B557, 413-442 (1999). [hep-th/9810035].

[30] J. Hoppe, V. Kazakov and I. K. Kostov, "Dimensionally reduced SYM(4) as solvable matrix quantum mechanics," Nucl. Phys. B 571, 479 (2000) hep-th/9907058.

[31] M. Hanada, M. Honda, Y. Honma, J. Nishimura, S. Shiba and Y. Yoshida, "Numerical studies of the ABJM theory for arbitrary N at arbitrary coupling constant," JHEP 1205, 121 (2012) arXiv:1202.5300 [hep-th]].

[32] G. T. Horowitz and A. Strominger, "Translations As Inner Derivations And Associativity Anomalies In Open String Field Theory," Phys. Lett. B 185, 45 (1987).

[33] E. Witten, "Noncommutative Geometry and String Field Theory," Nucl. Phys. B 268, 253 (1986).

[34] H. Hata and S. Moriyama, "Observables as twist anomaly in vacuum string field theory," JHEP 0201, 042 (2002) hep-th/0111034.

[35] L. Rastelli, A. Sen and B. Zwiebach, "Vacuum string field theory," hep-th/0106010.

[36] L. Rastelli, A. Sen and B. Zwiebach, "Star algebra spectroscopy," JHEP 0203, 029 (2002) hep-th/0111281.

[37] R. Dijkgraaf, R. Gopakumar, H. Ooguri and C. Vafa, "Baby universes in string theory," Phys. Rev. D 73, 066002 (2006) [hep-th/0504221]. 
[38] P. Dorey and R. Tateo, "Anharmonic oscillators, the thermodynamic Bethe ansatz, and nonlinear integral equations," J. Phys. A A 32, L419 (1999) hep-th/9812211.

[39] V. V. Bazhanov, S. L. Lukyanov and A. B. Zamolodchikov, "Spectral determinants for Schrodinger equation and Q operators of conformal field theory," J. Statist. Phys. 102, 567 (2001) hep-th/9812247].

[40] A. Voros, "Spectral Zeta Functions," Adv. Stud. Pure Math. 21 (1992) 327-358.

[41] A. Voros, "Exact quantization condition for anharmonic oscillators (in one dimension)," J. Phys. A A 27 (1994) 4653-4661.

[42] A. Voros, "Airy function (exact WKB results for potentials of odd degree)," J. Phys. A A 32 (1999) 1301-1311 arXiv:math-ph/9811001.

[43] V. V. Bazhanov, S. L. Lukyanov and A. B. Zamolodchikov, "Integrable structure of conformal field theory. 2. Q operator and DDV equation," Commun. Math. Phys. 190, 247 (1997) hep-th/9604044. 